\title{
Two-dimensional QCD in the Coulomb gauge
}

\author{
Yu.S.Kalashnikova*a ${ }^{* a}$ A.V.Nefediev ${ }^{\dagger a, b}$ \\ a Institute of Theoretical and Experimental Physics, \\ 117218, B.Cheremushkinskaya 25, Moscow, Russia \\ b Grupo Teórico de Altas Energias (GTAE), \\ Centro de Física das Interacções Fundamentais (CFIF), \\ Departamento de Física, Instituto Superior Técnico, \\ Av. Rovisco Pais, P-1049-001 Lisboa, Portugal
}

\begin{abstract}
In the present paper we discuss various aspects of the 't Hooft model for twodimensional QCD in the limit of infinite number of colours in the Coulomb gauge. The properties of mesonic excitations are addressed, with special attention paid to the pionic one. The twofold role of the pion is discussed: being a genuine $q \bar{q}$ state it is also a Goldstone boson of two-dimensional QCD. In particular, it is demonstrated explicitly how the soft-pion theorems are satisfied. It is pointed out that the Coulombgauge choice seems to be indispensable in studies of hadronic observables with the pions involved.
\end{abstract}

\section{Contents}

\begin{tabular}{llr}
\hline 1 & Introduction & 2
\end{tabular}

\begin{tabular}{|lll}
2 & Hamiltonian approach & 5
\end{tabular}

$2.1 \quad$ Dressed quarks and the mass-gap equation . . . . . . . . . . . . . . . . 6

2.2 The vacuum energy. Chirally-symmetric and nonsymmetric vacua . . . . . . 9

2.3 Generalized Bogoliubov transformation and mesonic compound states . . . . 11

2.4 The bound-state equation and properties of the mesonic wave functions . . . 15

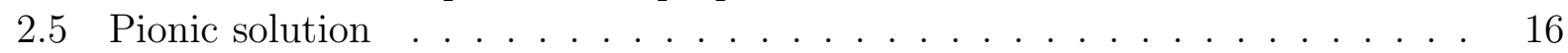

2.6 The one-particle limit and the nonpotential quark dynamics . . . . . . . . 16

2.7 A heavy-light system in the modified Fock-Schwinger gauge and the Lorentz nature of confinement . . . . . . . . . . . . . . . . 17

2.8 The chiral properties of the model in the Hamiltonian approach . . . . . . . 20

2.8 .1 The chiral condensate . . . . . . . . . . . . . . . . . . . . . . . . . . . . . . .

2.8 .2 The pion decay constant . . . . . . . . . . . . . . . . . . . . 23

2.8 .3 Partial conservation of the axial-vector current (PCAC) . . . . . . . . 24

*e-mail: yulia@heron.itep.ru

†e-mail: nefediev@heron.itep.ru nefediev@cfif.ist.utl.pt 
$\begin{array}{llr}3 & \text { Matrix approach } & 25\end{array}$

3.1 Matrix wave functions and matrix bound state equation . . . . . . . . . . . 25

3.2 Truncated Hilbert space and the problem of Hermiticity . . . . . . . . . . . 27

3.3 The chiral properties of the model in the matrix approach . . . . . . . . . . 29

3.3.1 The Gell-Mann-Oakes-Renner relation and the mass of the pion . . . 29

3.3 .2 The pionic solution beyond the chiral limit . . . . . . . . . . . 30

3.3 .3 The pion decay constant revisited . . . . . . . . . . . . . . . . . 31

3.4 Quark-quark scattering amplitude . . . . . . . . . . . . . . . . . . . . 32

3.5 Vector and axial-vector currents conservation. Ward identities. . . . . . . . . 34

3.6 The pionic vertex $\ldots \ldots \ldots \ldots \ldots \ldots$

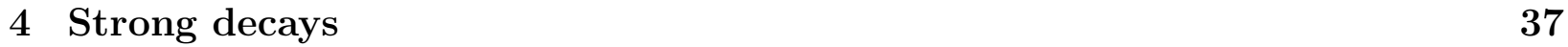

4.1 Suppressed terms in the Hamiltonian . . . . . . . . . . . . . . . . . . . 37

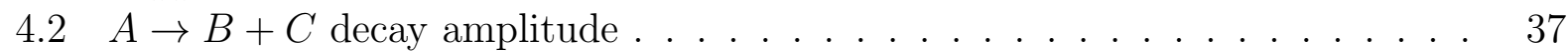

4.3 Adler selfconsistency condition $\ldots \ldots \ldots$. . . . . . . . . . . . . . . . . . . . 39

$\begin{array}{lll}5 \text { Conclusions and prospectives } & 40\end{array}$

$\begin{array}{lll}6 & \text { Appendix A } & 41\end{array}$

\section{Introduction}

Two-dimensional QCD in the limit $N_{C} \rightarrow \infty$ (the 't Hooft model [1]) was first considered in 70-th. Since then the 't Hooft model is widely used as a toy laboratory for studies of various aspects of strong interactions. The theory is relatively simple, as there are no transverse gluons in two dimensions; moreover, in the large- $N_{C}$ limit only planar graphs are to be summed up, and the theory is exactly solvable. Nevertheless, this is a truly relativistic field theory which does have a nontrivial content, resembling in such a way realistic $\mathrm{QCD}_{4}$ case. Indeed,

- the theory exhibits confinement and it is possible to demonstrate explicitly the existence of the discrete spectrum of the quark-antiquark bound states;

- the Poincaré invariance is maintained;

- the chiral symmetry is spontaneously broken;

- the Goldstone boson responsible for the chiral symmetry breaking is the $q \bar{q}$ ground state.

The first point is an almost trivial statement, since the Coulomb force is confining in two dimensions.

The second item is of a paramount significance for hadronic spectroscopy. It was demonstrated explicitly in [2] that, if a nonabelian theory is quantized in the explicitly noncovariant gauge, a special care should be taken of the Lorentz invariance. The quantum Poincaré algebra is closed only in the colour-singlet sector, which means that the spectrum can be evaluated in an arbitrary frame including, for example, the centre-of-mass frame as well as the infinite-momentum one. 
The chiral issue was historically a bit controversial. The initial studies in $\mathrm{QCD}_{2}$ were performed in the light-cone gauge. The pioneering paper [1] was followed by detailed studies of spectra and wave functions of mesons as well as hadronic interactions [3, 4]. A bit later an alternative approach was suggested, based on the Coulomb gauge $A_{1}=0$ [2] . The main advantage of the light-cone quantization is considerable simplification of the spectra calculations, but straightforward analysis gives the perturbative vacuum. The more technically involved version [2] yields a nontrivial vacuum, and it appears that a nonzero quark condensate exists for the massless quarks [5]. The latter feature is confirmed by the sum rule calculations in the light-cone gauge [6, 7]. At the hadronic level the apparent discrepancies are connected with a peculiar form of the pionic wave function near the chiral limit in the light-cone quantization, as it is discussed in [6], and will be explicitly demonstrated below. That is why one is forced to employ the sum rules and the Operator Product Expansion (OPE) to arrive at reliable results in the pionic physics and the vacuum structure [6]. On the contrary, the choice of the Coulomb gauge does not lead to drastic singularities and enables treating the pions on the same footing as other mesons. The conceptual difficulties of the light-cone quantization were resolved in the formulation on finite intervals [8], where the equivalence of both versions was demonstrated explicitly, clarifying the relationship between the light-cone and the equal-time quantization.

Since then a lot of work in two-dimensional QCD was done, employing the light-cone gauge. Among the questions discussed are the general properties of the OPE [6, 9] and heavy quark expansion and duality [10]. The calculations of spectra were performed in the framework of the so-called discretized light-cone quantization beyond the $N_{C} \rightarrow \infty$ limit [11]. A separate fascinating issue is the studies of $\mathrm{QCD}_{2}$ with adjoint fermions [12]. In the present paper we discuss the properties of vacuum and low-lying mesonic states built of light quarks in the Coulomb gauge, with special attention paid to the chiral issues of the theory.

Quantization on the light-cone allows one to establish an obvious connection with the dynamics of the parton model, while quantization on the ordinary time hypersurface is natural for another important branch of phenomenology, the constituent quark model. Indeed, the spectrum of $\mathrm{QCD}_{2}$ is discrete, and $N_{C} \rightarrow \infty$ limit suppresses additional quark pair creation, so that the 't Hooft model is nothing but a constituent quark model exactly derived from a nontrivial relativistic quantum field theory.

In the constituent quark models the confinement is usually modeled by a potential force. Then the gross features of the light quarkonia spectra and decays are described surprisingly well with an exception of the pion. Since one cannot include chiral symmetry breaking (CSB) effects into the constituent picture, there is no hope to reproduce the pion as the Goldstone boson, and soft pion theorems cannot be satisfied within any naive quark model picture.

The CSB phenomena follow from the most general symmetry considerations and have nothing to do with the particular mechanism of the confinement. One possible way to include the soft pions into the quark model is to introduce quarks and pions on equal footing, as it is done in the chiral quark models (see, e.g., 13] and references therein). Nevertheless, there are some results from the lattice simulations [14] demonstrating that the confinement and the CSB phenomena are present in the confining phase and disappear in unison above the deconfinement temperature; so both phenomena are interrelated dynamically.

A model was suggested many years ago [15], which connects the confinement and CSB (see also [16, where similar ideas were employed). The main ingredient of this model is an instantaneous three-dimensional oscillator confining force. Such a force does not follow from QCD and, in addition, there is no gauge and Lorentz invariance in this approach. An 
important development was suggested in [17], where the QCD vacuum is parametrized by a set of gauge and Lorentz invariant nonperturbative gluonic correlators which are responsible for both, the area law and the chiral condensate formation. The quark model which follows from such an approach should be able to reproduce, inter alia, all pion properties. In this regard it is instructive to study an exactly solvable theory with confinement and CSB, and the 't Hooft model is a perfect toy laboratory for such studies.

Before proceeding further we would like to note that the large- $N_{C}$ limit is essential in establishing the chiral properties of $\mathrm{QCD}_{2}$ [18]. The Coleman theorem [19] prohibits CSB for any finite number of degrees of freedom in a two-dimensional theory. It means that limits $N_{C} \rightarrow \infty$ and $m_{q} \rightarrow 0$ are not interchangeable. There is no contradiction with the Coleman theorem if one considers the weak coupling regime where $m_{q} \gg g \sim 1 / \sqrt{N_{C}}$, i.e., the limit of infinite number of colours is taken first (see [6] for the detailed discussion of this issue as well as of the other phase of the theory which corresponds to the strong coupling regime $\left.m_{q} \ll g\right)$.

The theory is given by the Lagrangian

$$
\begin{gathered}
L(x)=-\frac{1}{4} F_{\mu \nu}^{a}(x) F_{\mu \nu}^{a}(x)+\bar{q}(x)(i \hat{D}-m) q(x), \\
\hat{D}=\left(\partial_{\mu}-i g A_{\mu}^{a} t^{a}\right) \gamma_{\mu},
\end{gathered}
$$

and the large- $N_{C}$ limit implies that

$$
\gamma=\frac{g^{2} N_{C}}{4 \pi} \underset{N_{C} \rightarrow \infty}{\rightarrow} \text { const. }
$$

Our convention for $\gamma$ matrices is

$$
\gamma_{0} \equiv \beta=\sigma_{3}, \gamma_{1}=i \sigma_{2}, \gamma_{5} \equiv \alpha=\gamma_{0} \gamma_{1}=\sigma_{1} .
$$

The theory is gauged by the condition

$$
A_{1}\left(x_{0}, x\right)=0
$$

so that the only nontrivial correlator of the gluonic fields, the gluon propagator, takes the form

$$
\begin{gathered}
D_{01}^{a b}\left(x_{0}-y_{0}, x-y\right)=D_{11}^{a b}=\left(x_{0}-y_{0}, x-y\right)=0, \\
D_{00}^{a b}\left(x_{0}-y_{0}, x-y\right)=-\frac{i}{2} \delta^{a b}|x-y| \delta\left(x_{0}-y_{0}\right),
\end{gathered}
$$

and the infrared singularity is regularized by the principal value prescription, i.e., by means of an appropriate number of subtractions, for example,

$$
\begin{aligned}
\int \frac{d k}{(p-k)^{2}} F(k) & \rightarrow f \frac{d k}{(p-k)^{2}} F(k)= \\
& =\int \frac{d k}{(p-k)^{2}}\left(F(k)-F(p)-F^{\prime}(p)(k-p)-\ldots\right),
\end{aligned}
$$

yielding a linear confinement for the interquark interaction mediated by the two-dimensional gluon. 
(a)

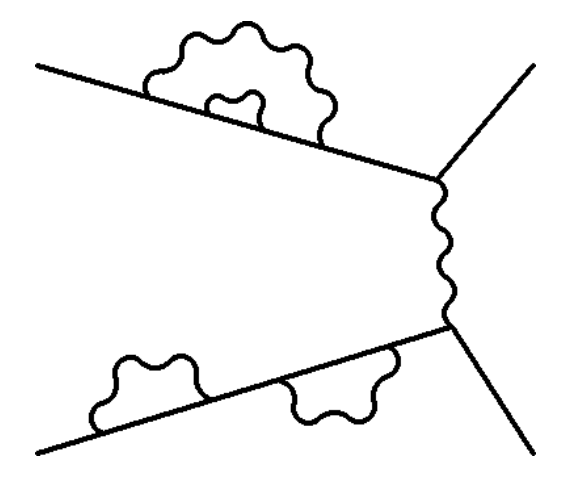

(b)

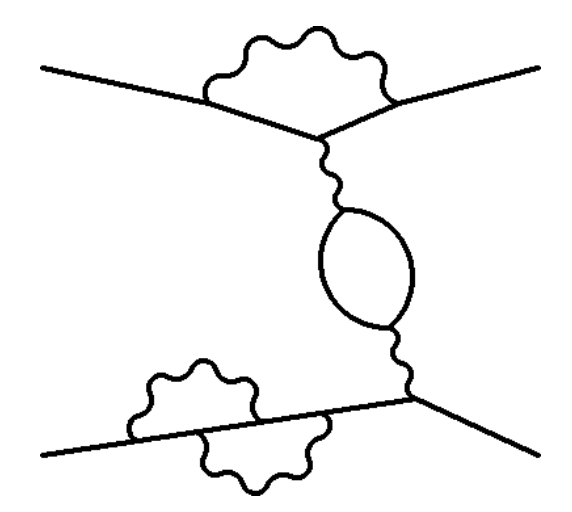

Figure 1: The planar (figure (a)) and the nonplanar, suppressed by $N_{C}$, (figure (b)) diagrams.

The paper is organized as follows. In Section 2 a Hamiltonian approach to $\mathrm{QCD}_{2}$ in the Coulomb gauge is developed. The bosonization of the model is performed explicitly in the large- $N_{C}$ limit, and a generalized Bogoliubov-Valatin transformation is employed to construct the composite operators creating and annihilating bosons. The pion wave function is found explicitly and the chiral properties of the theory are discussed. In Section 3 a matrix formalism is presented, a matrix bound-state equation is derived with special attention paid to the problem of the truncated Hilbert space and Hermiticity. We clarify the role of the backward motion of the $q \bar{q}$ pair in the meson. The current conservation and the Ward identities are discussed as well as the pionic vertex. Section 4 is devoted to strong hadronic decays, where we check the low-energy theorems, including the "Adler zero" selfconsistency condition. Concluding remarks are given in Section 5.

\section{Hamiltonian approach}

As is known from the pioneering work [1], the physical degrees of freedom in two-dimensional QCD in the weak-coupling regime are $q \bar{q}$ mesons. It would be quite natural then to reformulate the model entirely in terms of compound mesonic states, introducing a nonperturbative vacuum and creation and annihilation operators for the mesons, as excitations over this vacuum. The most natural framework for such a task is the Hamiltonian approach to the model, which we develop in this section.1 Note that it is possible in a selfconsistent form due to an instantaneous type of the interaction induced by (1.5).

This section is organized as follows. In subsection 2.1 we start from the Hamiltonian of the model in the Coulomb gauge, introduce dressed quark fields and, following [2], derive a gap equation (also known as mass-gap equation). We discuss the numerical solution to the gap equation found in [5]. Subsection 2.2 is devoted to investigation of the vacuum energy and identification of the chirally-symmetric and nonsymmetric vacua. In subsection 2.3 we introduce two-particle operators and perform a generalized Bololiubov transformation to diagonalize the Hamiltonian of the model in the mesonic sector. In subsection 2.4 we discuss properties and solutions of the bound-state equation, which appears as a consequence of

\footnotetext{
${ }^{1}$ The Hamiltonian approach to two-dimensional QCD in the light-cone gauge was developed in [20].
} 
the second Bogoliubov transformation. A special solution to the bound-state equation, the massless chiral pion, is found analytically and investigated in subsection 2.5. An issue connected to the locality and the Lorentz nature of confining interaction in two-dimensional QCD is discussed in subsections 2.6 and 2.7. In conclusion we turn to the chiral properties of the model and this is the subject of subsection 2.8 .

\subsection{Dressed quarks and the mass-gap equation}

Starting from the Lagrangian (1.1) and following the standard rules one arrives at the Hamiltonian of the theory in the form

$$
\begin{aligned}
H=\int d x q^{+}( & t, x)\left(-i \gamma_{5} \frac{\partial}{\partial x}+m \gamma_{0}\right) q(t, x)- \\
& -\frac{g^{2}}{2} \iint d x d y q^{+}(t, x) t^{a} q(t, x) q^{+}(t, y) t^{a} q(t, y) \frac{|x-y|}{2} .
\end{aligned}
$$

Note that only four-quark interaction enters the Hamiltonian (2.1) and this is a reflection of the fact stated in the Introduction that the only nontrivial gluonic correlator is the gluonic propagator (1.5). In four-dimensional QCD, possessing a much more complicated vacuum structure, correlators of all orders should appear, which gives rise to an infinite number of terms with four-quark, six-quark, etc interactions in the Hamiltonian. In the meantime, it is reasonable to truncate the $\mathrm{QCD}_{4}$ Hamiltonian at the four-quark interaction level, which corresponds to the bilocal approximation, when only $\langle A A\rangle$ correlator is left. The interested reader can find a detailed review of the given approach in [22].

The "dressed" quark field $q(x)$ in (2.1) is defined as follows [2]

$$
\begin{gathered}
q_{\alpha i}(t, x)=\int \frac{d k}{2 \pi} e^{i k x}\left[b_{\alpha}(k, t) u_{i}(k)+d_{\alpha}^{+}(-k, t) v_{i}(-k)\right], \\
b_{\alpha}(t, k)|0\rangle=d_{\alpha}(t,-k)|0\rangle=0, \quad b_{\alpha}^{+}(t, k)|0\rangle=|q\rangle, \quad d_{\alpha}^{+}(t,-k)|0\rangle=|\bar{q}\rangle, \\
\left\{b_{\alpha}(t, p) b_{\beta}^{+}(t, q)\right\}=\left\{d_{\alpha}(t,-p) d_{\beta}^{+}(t,-q)\right\}=2 \pi \delta(p-q) \delta_{\alpha \beta}, \\
u(k)=T(k)\left(\begin{array}{l}
1 \\
0
\end{array}\right), \quad v(-k)=T(k)\left(\begin{array}{l}
0 \\
1
\end{array}\right), \\
T(k)=e^{-\frac{1}{2} \theta(k) \gamma_{1}},
\end{gathered}
$$

where $\theta$ plays the role of the Bogoliubov-Valatin angle. Greek and Latin letters denote colour and spinor indices, respectively. Strong interaction between quarks implies that the true vacuum state is described by a nontrivial $\theta$, whereas excitations over it bring positive contribution to the energy.

In what follows we shall omit the explicit dependence of operators on time. It can be easily restored at any intermediate step, thus giving, for example,

$$
b_{\alpha}(t, p)=b_{\alpha}(p) e^{-i E(p) t}, \quad d_{\alpha}(t,-p)=d_{\alpha}(-p) e^{i E(p) t},
$$

where $E(p)$ is the dispersive law of the dressed quark (to be defined later).

\footnotetext{
${ }^{2}$ These are irreducible averages (cumulants) meant here, which are defined as $\langle\langle O\rangle\rangle=\langle O\rangle,\left\langle\left\langle O_{1} O_{2}\right\rangle\right\rangle=$ $\left\langle O_{1} O_{2}\right\rangle-\left\langle O_{1}\right\rangle\left\langle O_{2}\right\rangle$, etc 21 . Due to the Lorentz and the colour invariances of the QCD vacuum $\left\langle A_{\mu}^{a}\right\rangle=0$ and, hence, $\left\langle\left\langle A_{\mu}^{a} A_{\nu}^{b}\right\rangle\right\rangle=\left\langle A_{\mu}^{a} A_{\nu}^{b}\right\rangle$.
} 
The Hamiltonian (2.1) normally ordered in the basis (2.3) splits into three parts $(L$ is the one-dimensional volume),

$$
H=L N_{C} \mathcal{E}_{v}+: H_{2}:+: H_{4}:
$$

where

$$
\mathcal{E}_{v}=\int \frac{d p}{2 \pi} \operatorname{Tr}\left\{\left(\gamma_{5} p+m \gamma_{0}\right) \Lambda_{-}(p)+\frac{\gamma}{4 \pi} \int \frac{d k}{(p-k)^{2}} \Lambda_{+}(k) \Lambda_{-}(p)\right\}
$$

is the vacuum energy density,

$$
\begin{aligned}
: H_{2}:= & \int d x: q_{i}^{+}(x)\left(-i \gamma_{5} \frac{\partial}{\partial x}+m \gamma_{0}\right) q_{i}(x):- \\
& -\frac{\gamma}{2} \int d x d y \frac{|x-y|}{2} \int d k: q_{i}^{+}(x)\left[\Lambda_{+}(k)-\Lambda_{-}(k)\right] q_{i}(y): e^{i k(x-y)}
\end{aligned}
$$

is quadratic in quark fields, whereas the $: H_{4}:$ part contains four of them,

$$
: H_{4}:=-\frac{g^{2}}{2} \int d x d y: q^{+}(x) t^{a} q(x) q^{+}(y) t^{a} q(y): \frac{|x-y|}{2} .
$$

We have introduced projectors onto positive and negative states for convenience:

$$
\Lambda_{ \pm}(p)=T(p) \frac{1 \pm \gamma_{0}}{2} T^{+}(p)
$$

One comment on the role played by different parts of the Hamiltonian (2.7) is in order. The first term in (2.7) defines the energy of the vacuum which is to be minimized. The : $H_{2}$ : part describes "dressing" of quarks, so that an alternative approach to minimizing $\mathcal{E}_{v}$ is the requirement that : $H_{2}$ : be diagonal in terms of the quark creation and annihilation operators, or, equivalently, that the anomalous Bogoliubov term be absent. No matter which way is used, the resulting equations for the Bogoliubov-Valatin angle $\theta$ and the dispersive law for the dressed quarks read [2]:

$$
\left\{\begin{array}{l}
E(p) \cos \theta(p)=m+\frac{\gamma}{2} f \frac{d k}{(p-k)^{2}} \cos \theta(k) \\
E(p) \sin \theta(p)=p+\frac{\gamma}{2} f \frac{d k}{(p-k)^{2}} \sin \theta(k),
\end{array}\right.
$$

which can be reformulated in the form of the gap equation for the angle $\theta$,

$$
p \cos \theta(p)-m \sin \theta(p)=\frac{\gamma}{2} f \frac{d k}{(p-k)^{2}} \sin [\theta(p)-\theta(k)]
$$

If a solution for $\theta$ is known, $E(p)$ can be easily found from the relation

$$
E(p)=m \cos \theta(p)+p \sin \theta(p)+\frac{\gamma}{2} f \frac{d k}{(p-k)^{2}} \cos [\theta(p)-\theta(k)]
$$

The gap equation (2.13) is subject to numerical investigation which was performed in [5]. The results for $\theta(p)$ and $E(p)$ are given in Fig.2.

Notice several important properties of the functions $\theta$ and $E$ : 

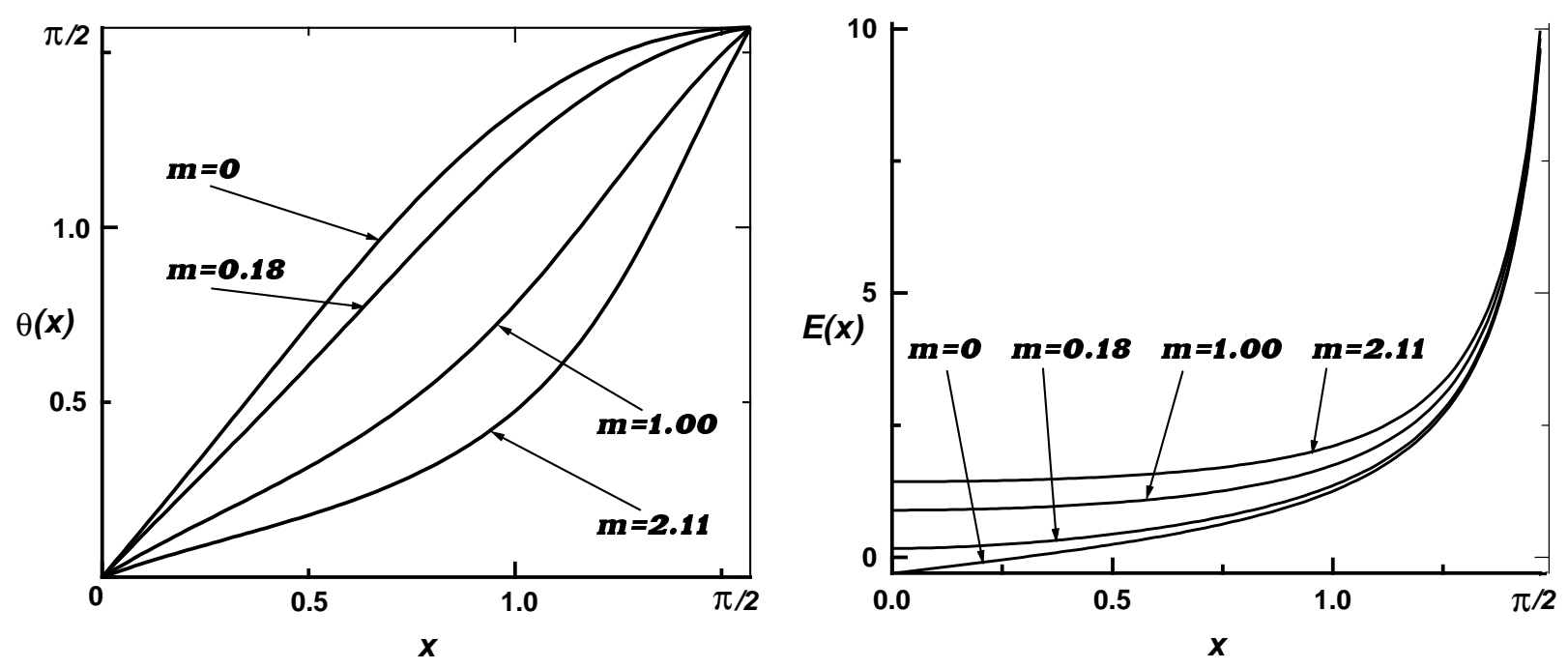

Figure 2: Numerical solutions for the Bogoliubov angle $\theta(p)$ and the dressed quark dispersive law $E(p)$ for different values of the quark mass. The plots are taken from [5], where $x$ comes from the change of variable $p=\tan (x)$, all dimensional quantities are given in the proper units of $(2 \gamma)^{1 / 2}$.

- $\theta(p)$ is odd, whereas $E(p)$ is even,

$$
\theta(-p)=-\theta(p), \quad E(-p)=E(p)
$$

- solution for $\theta(p)$ remains nontrivial even in the chiral limit, $m=0$, and so does the solution for the dispersive law (see Fig.2);

- the function $E(p)$ is not positively defined, as it might be naively expected (see Fig.2).

The formal reason for the last property comes from the regularization prescription (1.6), which defines the way of subtracting an infinite self-energy constant. Thus a divergent integral of positively defined functions might lead to a negative result after the proper regularization.

One can easily check that

$$
\theta(p \rightarrow 0) \sim p, \quad \theta^{\prime}(0)>0
$$

and

$$
E(0)=m+\frac{\gamma}{2} f \frac{d k}{k^{2}} \cos \theta(k)=m-\frac{\gamma}{2} \int_{0}^{\infty} \frac{d k}{k^{2}} \sin ^{2} \frac{\theta(k)}{2} \approx m-\frac{\pi \gamma}{8} \theta^{\prime}(0),
$$

so that $E(0)$ becomes negative for $m$ smaller than some critical value $m_{c}$. This does not lead to a disaster as, according to findings of [2], only the colour-singlet sector of the theory is Lorentz covariant, whereas the quark sector is not colour-singlet and, hence, it may not be Lorentz covariant. Nevertheless, one has to be especially careful shifting the poles in the quark propagator, paying attention to the sign of the product of $E(p)$ and the infinitely small positive constant $\varepsilon$ [2]. 
Note that a simple analytical solution to the gap equation (2.13) in the chiral limit was found in [2], which reads]

$$
\theta(p)=\frac{1}{2} \pi \operatorname{sign}(p), \quad E(p)=|p|-P \frac{\gamma}{|p|},
$$

where the symbol $P$ stands for the principal value prescription. It is clearly seen from (2.18) that for this solution $E(p)$ is not positively defined either.

The two solutions to the gap equation in the chiral limit, the one given by (2.18) and the other, depicted in Fig.2, define two different phases of the theory. The chiral symmetry is broken in the latter case only, whereas in the former one it remains unbroken.

It was demonstrated in [23] that the analytical solution (2.18) possesses infinite energy compared to the numerical chirally-nonsymmetric one given in Fig.2. Thus the chiral symmetry is never restored in the 't Hooft model and there is only one phase of the theory with chiral symmetry spontaneously broken (see the next subsection for the details). Such way, the solution (2.18) is unphysical, but we still prefer to keep it to exemplify some statements concerning the chiral properties of the model. In the next subsection we discuss this issue in more detail.

Performing all necessary calculations with the precautions discussed above, one arrives at the Hamiltonian of the model which is diagonal with respect to the dressed quarks basis. The contribution of the : $H_{4}$ : part of the Hamiltonian (2.7) is suppressed by an extra factor $1 / \sqrt{N_{C}}$ and thus it can be neglected in the single-quark sector of the theory;

$$
: H:=L N_{C} \mathcal{E}_{v}+\int \frac{d k}{2 \pi} E(k)\left\{b_{\alpha}^{+}(k) b_{\alpha}(k)+d_{\alpha}^{+}(-k) d_{\alpha}(-k)\right\} .
$$

Note that the result (2.19) itself has practically no value as it deals with the gaugeand Lorentz-noncovariant sector. The most important result of this subsection is the gap equation (2.13) and the numerical solution to it depicted in Fig.2. They will be intensively used in what follows.

\subsection{The vacuum energy. Chirally-symmetric and nonsymmetric vacua}

Let us return to the gap equation (2.13) and discuss an alternative way of its derivation namely, the minimization of the vacuum energy (2.8) [23]. We consider the case of massless quarks, $m=0$.

It is convenient to introduce an excess of the vacuum energy density for the theory with interaction over the free-theory one,

$$
\Delta \mathcal{E}_{v}[\theta]=\mathcal{E}_{v}[\theta]-\mathcal{E}_{v}\left[\theta_{\text {free }}\right]=-\int \frac{d p}{2 \pi}(p \sin \theta(p)-|p|)-\frac{\gamma}{4 \pi} \int \frac{d p d k}{(p-k)^{2}} \cos [\theta(p)-\theta(k)]
$$

where $\theta_{\text {free }}(p)=\frac{\pi}{2} \operatorname{sign}(p)$ corresponds to the free $(\gamma=0)$ massless theory.

The gap equation, following from the minimization procedure,

$$
\frac{\delta}{\delta \theta(p)} \Delta \mathcal{E}_{v}[\theta]=0
$$

\footnotetext{
${ }^{3}$ In fact, any odd function $\theta(p)$ with an arbitrary number of jumps from $\pi / 2$ to $-\pi / 2$ and back also satisfies the gap equation (2.13) [2]. Meanwhile solutions of such a type do not reduce to the free theory when $\gamma$ tends to zero and they will not be discussed.
} 
readily reproduces equation (2.13).

To ensure that the solution to equation (2.13) indeed minimizes the vacuum energy we use the following approach [23]. Let $\theta(p)$ be the solution to (2.21) corresponding to the minimum of $\Delta \mathcal{E}_{v}$. Then $\theta(p / A)$ stretched with an arbitrary parameter $A$ should enlarge the energy (2.20). Naive dimensional analysis demonstrates that $\Delta \mathcal{E}_{v}$ scales with $A$ as

$$
\Delta \mathcal{E}_{v}=\frac{1}{2} C_{1} A^{2}-\gamma C_{2},
$$

with $C_{1,2}$ being positive constants. Then the stable solution is provided by $A_{0}$ minimizing the energy (2.22), i.e., by $A_{0}=0$, which corresponds either to the free massless theory, giving $\Delta \mathcal{E}_{v}=0$, or to the analytic solution (2.18). Both solutions correspond to the preserved chiral symmetry. Thus one arrives at a discouraging conclusion that there is no nontrivial chirallynonsymmetric solution to the gap equation (2.13). In the meantime, the naive analysis performed above completely ignores the fact that the vacuum energy (2.20) is logarithmically infrared divergent due to the second term. Introducing a cut-off and repeating the same steps, one can conclude that the correct dependence of the vacuum energy on the scale parameter $A$ is as follows:

$$
\Delta \mathcal{E}_{v}=\frac{1}{2} C_{1} A^{2}-\gamma C_{2} \ln A+\gamma C_{3}
$$

instead of the naive form (2.22). The constant $C_{3}$ is proportional to the logarithm of the cut-off and can be removed by an infinite renormalization.

Note that the second term in (2.20) cannot be made convergent both, in the infrared and in the ultraviolet simultaneously. Indeed, one can remove the infrared divergence in (2.20), subtracting the energy corresponding to the solution (2.18) instead of the free one. Then the resulting integral appears ultraviolet logarithmically divergent and leads to the same relation (2.23) but with the infinite constant $C_{3}$ containing the logarithm of the ultraviolet cut-off.

The function (2.23) always has a minimum at

$$
A_{0}=\sqrt{\gamma \frac{C_{2}}{C_{1}}},
$$

which corresponds to the nontrivial solution of the gap equation found numerically in [5] and depicted in Fig.2. In the meantime, from the form of the function (2.23) one can see the logarithmic growth of the energy in approaching the solution (2.18), which, as was discussed above, corresponds to $A_{0}=0$.

Thus ones arrives at the conclusion, already mentioned above, that the vacuum energy corresponding to the chirally-symmetric solution (2.18) is infinite compared to that for the chirally-nonsymmetric one depicted in Fig. 22 and, hence, no phase transition of the chiral symmetry restoration is possible in the 't Hooft model.

It is instructive to note that the very fact of the existence of the chirally-nonsymmetric nontrivial solution to the gap equation (2.13) is yet another consequence of the infrared behaviour of the 't Hooft model discussed above. Indeed, the gap equation (2.13) was derived

\footnotetext{
${ }^{4}$ Once the logarithmically divergent term in $(2.23)$ is proportional to the coupling constant $\gamma$, then there is no problem with the free limit of the theory, which also corresponds to $A_{0}=0$. Indeed, when $\gamma$ tends to zero the logarithmic term in (2.23) disappears, so that, as defined by (2.20), the vacuum energy of the free theory is zero.
} 
in neglection of all effects of the fermionic vacuum polarization (creation and annihilation fermionic operators introduced in (2.3) correspond to the so-called BCS approximation). In the meantime, the chiral symmetry can be spontaneously broken in other two-dimensional models for QCD, like the Gross-Neveu one [24, 25], but in order to have a nonzero chiral condensate one has to go beyond BCS level, summing up fermionic bubbles, whereas the equation similar to (2.13) has only a trivial chirally-symmetric solution, giving $\langle\bar{q} q\rangle=0$.

\subsection{Generalized Bogoliubov transformation and mesonic compo- und states}

In the previous subsection the first two terms of the Hamiltonian (2.7) were considered. Let us turn to the third part, : $H_{4}$ :. With substituted solution for the dressed quarks, it describes interaction between them, which leads to formation of the $q \bar{q}$ bound states mesons. In this subsection we are to diagonalize the Hamiltonian (2.7) in the colour-singlet mesonic sector of the theory. To this end we introduce compound operators which act on colourless pairs of quarks and antiquarks [8, 26]:

$$
\begin{gathered}
B\left(p, p^{\prime}\right)=\frac{1}{\sqrt{N_{C}}} b_{\alpha}^{+}(p) b_{\alpha}\left(p^{\prime}\right), \quad D\left(p, p^{\prime}\right)=\frac{1}{\sqrt{N_{C}}} d_{\alpha}^{+}(-p) d_{\alpha}\left(-p^{\prime}\right), \\
M\left(p, p^{\prime}\right)=\frac{1}{\sqrt{N_{C}}} d_{\alpha}(-p) b_{\alpha}\left(p^{\prime}\right), \quad M^{+}\left(p, p^{\prime}\right)=\frac{1}{\sqrt{N_{C}}} b_{\alpha}^{+}\left(p^{\prime}\right) d_{\alpha}^{+}(-p),
\end{gathered}
$$

with the commutation relations being

$$
\begin{gathered}
{\left[M\left(p, p^{\prime}\right) M^{+}\left(q, q^{\prime}\right)\right]=(2 \pi)^{2} \delta\left(p^{\prime}-q^{\prime}\right) \delta(p-q)-} \\
-\frac{2 \pi}{\sqrt{N_{C}}}\left\{D(q, p) \delta\left(p^{\prime}-q^{\prime}\right)+B\left(q^{\prime}, p^{\prime}\right) \delta(p-q)\right\}_{N_{C} \rightarrow \infty}^{\rightarrow} \\
\rightarrow(2 \pi)^{2} \delta\left(p^{\prime}-q^{\prime}\right) \delta(p-q), \\
{\left[B\left(p, p^{\prime}\right) B\left(q, q^{\prime}\right)\right]=\frac{2 \pi}{\sqrt{N_{C}}}\left(B\left(p, q^{\prime}\right) \delta\left(p^{\prime}-q\right)-B\left(q, p^{\prime}\right) \delta\left(p-q^{\prime}\right)\right)_{N_{C} \rightarrow \infty}^{\rightarrow} 0,} \\
{\left[D\left(p, p^{\prime}\right) D\left(q, q^{\prime}\right)\right]=\frac{2 \pi}{\sqrt{N_{C}}}\left(D\left(p, q^{\prime}\right) \delta\left(p^{\prime}-q\right)-D\left(q, p^{\prime}\right) \delta\left(p-q^{\prime}\right)\right)_{N_{C} \rightarrow \infty}^{\rightarrow} 0 .} \\
{\left[B\left(p, p^{\prime}\right) M\left(q, q^{\prime}\right)\right]=-\frac{2 \pi}{\sqrt{N_{C}}} M^{\rightarrow}\left(q, p^{\prime}\right) \delta\left(p-q^{\prime}\right) \underset{N_{C} \rightarrow \infty}{\rightarrow} 0,} \\
{\left[B\left(p, p^{\prime}\right) M^{+}\left(q, q^{\prime}\right)\right]=\frac{2 \pi}{\sqrt{N_{C}}} M^{+}(q, p) \delta\left(p^{\prime}-q^{\prime}\right) \underset{N_{C} \rightarrow \infty}{\rightarrow} 0,} \\
{\left[D\left(p, p^{\prime}\right) M^{\rightarrow}\left(q, q^{\prime}\right)\right]=-\frac{2 \pi}{\sqrt{N_{C}}} M^{\rightarrow}\left(p^{\prime}, q^{\prime}\right) \delta(p-q) \underset{N_{C} \rightarrow \infty}{\rightarrow} 0,} \\
{\left[D\left(p, p^{\prime}\right) M^{+}\left(q, q^{\prime}\right)\right]=\frac{2 \pi}{\sqrt{N_{C}}} M^{+}\left(p, q^{\prime}\right) \delta\left(p^{\prime}-q\right)_{N_{C} \rightarrow \infty}^{\rightarrow} 0 .}
\end{gathered}
$$


With the new operators substituted, the Hamiltonian (2.7) takes the form

$$
\begin{gathered}
H=L N_{C} \mathcal{E}_{v}+\sqrt{N_{C}} \int \frac{d k}{2 \pi} E(k)\{B(k, k)+D(k, k)\} \\
-\frac{\gamma}{2} \int \frac{d p d k d Q}{(2 \pi)^{2}(p-k)^{2}}\left[2 \cos \frac{\theta(p)-\theta(k)}{2} \sin \frac{\theta(Q-p)-\theta(Q-k)}{2}\right. \\
\times\left\{M^{+}(p, p-Q) D(k-Q, k)+M^{+}(p-Q, p) B(k-Q, k)\right. \\
-B(p, p-Q) M(k-Q, k)-D(p, p-Q) M(k, k-Q)\} \\
\times\{B(p-Q, p) B(k, k-Q)+D(p, p-Q) D(k-Q, k) \\
\left.+M^{+}(p-Q, p) M(k-Q, k)+M^{+}(p, p-Q) M(k, k-Q)\right\} \\
\quad+\sin \frac{\theta(p)-\theta(k)}{2} \sin \frac{\theta(Q-p)-\theta(Q-k)}{2} \cos \frac{\theta(Q-p)-\theta(Q-k)}{2} \\
\times\{B(p, p-Q) D(k, k-Q)+B(p-Q, p) D(k-Q, k) \\
\left.\left.+M(p, p-Q) M(k-Q, k)+M^{+}(p-Q, p) M^{+}(k, k-Q)\right\}\right]
\end{gathered}
$$

where : $H_{2}$ : and : $H_{4}$ : terms should have the same order in powers of $N_{C}$ and thus act together as opposed to the one-body sector, where : $H_{4}$ : was suppressed as $1 / \sqrt{N_{C}}$.

A crucial step we are to perform now is to note, that in the mesonic sector of the theory one cannot create and annihilate isolated quarks and antiquarks. Only colourless $q \bar{q}$ pairs can appear, so that, creating a quark, we have to create an accompanying antiquark and, vice versa, for each created antiquark we have an extra quark. Thus the operators (2.25) cannot be independent. Indeed, it is easy to check that the substitution

$$
\begin{aligned}
& B\left(p, p^{\prime}\right)=\frac{1}{\sqrt{N_{C}}} \int \frac{d q^{\prime \prime}}{2 \pi} M^{+}\left(q^{\prime \prime}, p\right) M\left(q^{\prime \prime}, p^{\prime}\right), \\
& D\left(p, p^{\prime}\right)=\frac{1}{\sqrt{N_{C}}} \int \frac{d q^{\prime \prime}}{2 \pi} M^{+}\left(p, q^{\prime \prime}\right) M\left(p^{\prime}, q^{\prime \prime}\right)
\end{aligned}
$$

satisfies the commutation relations (2.26). Now one can neglect a number of terms in the Hamiltonian (2.27) and to arrive at a simplified expression,

$$
\begin{gathered}
H=L N_{C} \mathcal{E}_{v}+\int \frac{d Q d p}{(2 \pi)^{2}}\left[(E(p)+E(Q-p)) M^{+}(p-Q, p) M(p-Q, p)\right. \\
-\frac{\gamma}{2} \int \frac{d k}{(p-k)^{2}}\left\{2 C(p, k, Q) M^{+}(p-Q, p) M(k-Q, k)\right. \\
\left.\left.+S(p, k, Q)\left(M(p, p-Q) M(k-Q, k)+M^{+}(p, p-Q) M^{+}(k-Q, k)\right)\right\}\right],
\end{gathered}
$$


where

$$
\begin{aligned}
& C(p, k, Q)=\cos \frac{\theta(p)-\theta(k)}{2} \cos \frac{\theta(Q-p)-\theta(Q-k)}{2}, \\
& S(p, k, Q)=\sin \frac{\theta(p)-\theta(k)}{2} \sin \frac{\theta(Q-p)-\theta(Q-k)}{2} .
\end{aligned}
$$

The operators $M^{+}$and $M$ create and annihilate quark-antiquark pairs, which are not mesons yet since Hamiltonian (2.29) is not diagonal in terms of these operators.

Symbolically the operator structure of the Hamiltonian (2.29),

$$
H \sim H_{0}+A M^{+} M+\frac{1}{2} B\left(M^{+} M^{+}+M M\right),
$$

resembles the one appearing in the theory of Bose gas with interaction, where the last term on the r.h.s. describes interaction between particles and leads to appearing of quasiparticles diagonalizing the Hamiltonian. Thus (2.29) is subject to another Bogoliubov transformation. Once operators $M^{+}$and $M$ obey the Bose statistics, then the general form of the transformation is expected to be

$$
\left\{\begin{array}{l}
m=u M+v M^{+} \\
m^{+}=u M^{+}+v M,
\end{array}\right.
$$

with $u$ and $v$ obeying the standard bosonic condition,

$$
u^{2}-v^{2}=1 .
$$

Of course, one needs to generalize transformation (2.32), (2.33) in order to take into account the nonlocality of the involved objects. Such a generalization takes the form [26]

$$
\begin{gathered}
m_{n}^{+}(Q)=\int \frac{d q}{2 \pi}\left\{M^{+}(q-Q, q) \varphi_{+}^{n}(q, Q)+M(q, q-Q) \varphi_{-}^{n}(q, Q)\right\}, \\
m_{n}(Q)=\int \frac{d q}{2 \pi}\left\{M(q-Q, q) \varphi_{+}^{n}(q, Q)+M^{+}(q, q-Q) \varphi_{-}^{n}(q, Q)\right\}, \\
M(p-P, p)=\sum_{n>0}\left\{m_{n}(P) \varphi_{+}^{n}(p, P)-m_{n}^{+}(P) \varphi_{-}^{n}(p, P)\right\}, \\
M^{+}(p-P, p)=\sum_{n>0}\left\{m_{n}^{+}(P) \varphi_{+}^{n}(p, P)-m_{n}(P) \varphi_{-}^{n}(p, P)\right\},
\end{gathered}
$$

where $\varphi_{ \pm}$stand for Bogoliubov-like functions $u$ and $v$, so it is not surprise that they obey the following orthonormality and completeness conditions, which are nothing but the generalization of (2.33):

$$
\begin{gathered}
\int \frac{d p}{2 \pi}\left(\varphi_{+}^{n}(p, Q) \varphi_{+}^{m}(p, Q)-\varphi_{-}^{n}(p, Q) \varphi_{-}^{m}(p, Q)\right)=\delta_{n m}, \\
\int \frac{d p}{2 \pi}\left(\varphi_{+}^{n}(p, Q) \varphi_{-}^{m}(p, Q)-\varphi_{-}^{n}(p, Q) \varphi_{+}^{m}(p, Q)\right)=0, \\
\sum_{n=0}^{\infty}\left(\varphi_{+}^{n}(p, Q) \varphi_{+}^{n}(k, Q)-\varphi_{-}^{n}(p, Q) \varphi_{-}^{n}(k, Q)\right)=2 \pi \delta(p-k), \\
\sum_{n=0}^{\infty}\left(\varphi_{+}^{n}(p, Q) \varphi_{-}^{n}(k, Q)-\varphi_{-}^{n}(p, Q) \varphi_{+}^{n}(k, Q)\right)=0 .
\end{gathered}
$$



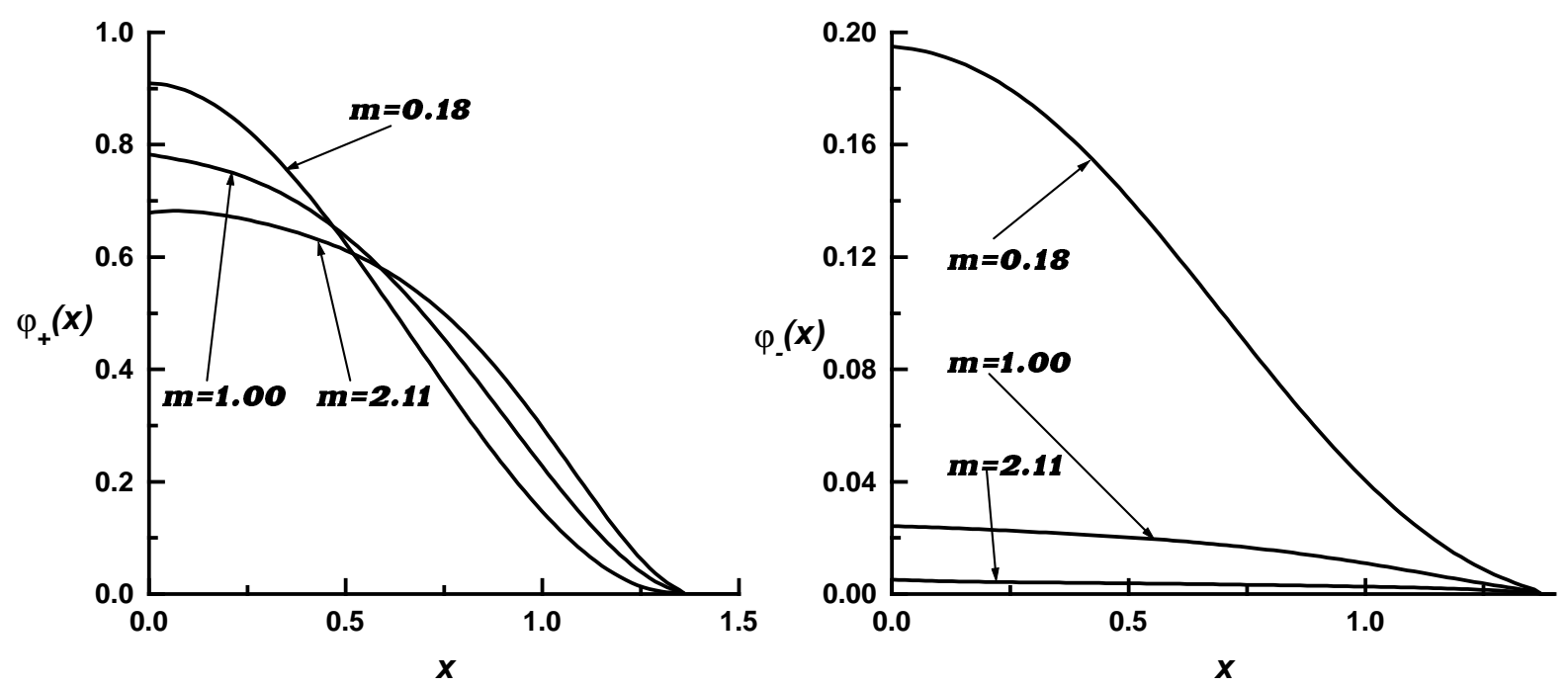

Figure 3: The $\varphi_{+}$and $\varphi_{-}$components of the ground-state wave function in the meson rest frame for different masses of the quark taken from [5]. Note that $\varphi_{ \pm}$are even functions of $p$ for the ground state. The variable $x$ comes from the change $p=\tan (x)$, all dimensional quantities are given in the proper units of $(2 \gamma)^{1 / 2}$.

The functions $\varphi_{ \pm}^{n}$ play the role of the meson wave functions, moreover, one can easily establish the physical meaning of both. Namely, $\varphi_{+}^{n}$ describes the motion forward in time of the $q \bar{q}$ pair inside meson, whereas $\varphi_{-}^{n}$ is responsible for its motion backward in time. We shall return to this issue later on when discussing the properties of the bound-state equation.

The physical meaning of the transformation (2.34) is quite obvious. Indeed, in the theory with a nontrivial vacuum there are two ways to produce a quark-antiquark bound state. The first way, which works no matter if the vacuum is trivial or not, is to create the $q \bar{q}$ pair directly, by means of the operator $M^{+} \sim b^{+} d^{+}$. In the meantime, if the vacuum is nontrivial and contains the chiral condensate $\langle\bar{q} q\rangle \neq 0$, then one can "borrow" a finite number of correlated quark-antiquark pairs from the vacuum and to remove redundant particles, using the annihilation operator $M \sim d b$. The wave functions $\varphi_{ \pm}$describe the contributions of these two procedures. It follows immediately from such a consideration that, e.g., for the case of massive quarks $\varphi_{-}$should be small since the condensate of heavy quarks is suppressed by the large quark mass.

It is easy to check that operators (2.34) obey the standard bosonic commutation relations,

$$
\begin{gathered}
{\left[m_{n}(Q) m_{m}^{+}\left(Q^{\prime}\right)\right]=2 \pi \delta\left(Q-Q^{\prime}\right) \delta_{n m},} \\
{\left[m_{n}(Q) m_{m}\left(Q^{\prime}\right)\right]=\left[m_{n}^{+}(Q) m_{m}^{+}\left(Q^{\prime}\right)\right]=0,}
\end{gathered}
$$

and diagonalize the Hamiltonian (2.29),

$$
H=L N_{C} \mathcal{E}_{v}^{\prime}+\sum_{n=0}^{+\infty} \int \frac{d Q}{2 \pi} Q_{n}^{0}(Q) m_{n}^{+}(Q) m_{n}(Q),
$$

if the wave functions $\varphi_{ \pm}^{n}$ are solutions to the bound-state equation which we discuss in the next subsection. The vacuum energy in (2.39) contains extra contributions compared to that 
in (2.29), besides that the vacuum structure itself is changed, so that the real vacuum of the theory, $|\Omega\rangle$, differs from $|0\rangle$ introduced in (2.3) and they are related through a unitary transformation.

\subsection{The bound-state equation and properties of the mesonic wave functions}

As in case of the first Bogoliubov transformation performed in subsection 2.1, when the gap equation (2.13) appeared as a condition of the Hamiltonian diagonalization, the second, generalized, Bogoliubov transformation described in the previous subsection also leads to an equation defining the Bogoliubov-like functions $\varphi_{ \pm}^{n}$. This is nothing but the bound-state equation for the mesonic spectrum of the model [2]|尸:

$$
\left\{\begin{aligned}
{[E(p)} & \left.+E(Q-p)-Q_{0}\right] \varphi_{+}(Q, p)= \\
& =\gamma f \frac{d k}{(p-k)^{2}}\left[C(p, k, Q) \varphi_{+}(Q, k)-S(p, k, Q) \varphi_{-}(Q, k)\right] \\
{[E(p)} & \left.+E(Q-p)+Q_{0}\right] \varphi_{-}(Q, p)= \\
& =\gamma f \frac{d k}{(p-k)^{2}}\left[C(p, k, Q) \varphi_{-}(Q, k)-S(p, k, Q) \varphi_{+}(Q, k)\right] .
\end{aligned}\right.
$$

Unfortunately, analytical investigation of the system (2.40) is possible only in some specific cases (we discuss one of them in the next subsection), so that it is rather subject to numerical studies. In Fig.3 taken from paper [5] we give numerical solutions for the ground state for different quark masses. It is clearly seen that the $\varphi_{-}$component of the mesonic wave function is suppressed for large masses of the quark in full agreement with the consideration presented above. It is also suppressed for highly excited states [5]. Indeed, the case of higher excitations is very close to the quasiclassical regime, where the answers for the spectra coincide with those of the quantum mechanical problem of the two-dimensional string with quarks at the ends [27], which, in turn, is reduced to a quark potential model.

Let us demonstrate how the celebrated 't Hooft equation comes out from (2.40) [2]. In the above mentioned paper the Lorentz covariance was proved for the colour-singlet sector of the theory, so that the spectrum of mass of the $q \bar{q}$ bound states, following from (2.40), should not change when one performs boosts, even for the limiting case of the boost into the infinite-momentum frame, $P \rightarrow \infty$. One can easily verify that in this case $S(p, k, Q) \rightarrow 0$, whereas $C(p, k, Q)$ turns into a step-like function, so that the region of integration on the r.h.s. of (2.40) shrinks to a finite interval, $0<x<1$, with $x=p / P$ being the share of the total momentum carried by the quark (the 't Hooft variable). The $\varphi_{-}$component of the

\footnotetext{
${ }^{5}$ It is straightforward to generalize the bound-state equation (2.40) for the case of a many-flavour theory. One needs to make the following modifications:

$$
\begin{gathered}
E(p) \rightarrow E_{f_{1}}(p), \quad E(Q-p) \rightarrow E_{f_{2}}(Q-p), \quad \varphi_{ \pm} \rightarrow \varphi_{ \pm}^{f_{1} f_{2}} \\
C(p, k, Q) \rightarrow C_{f_{1} f_{2}}(p, k, Q)=\cos \frac{\theta_{f_{1}}(p)-\theta_{f_{1}}(k)}{2} \cos \frac{\theta_{f_{2}}(Q-p)-\theta_{f_{2}}(Q-k)}{2} \\
S(p, k, Q) \rightarrow S_{f_{1} f_{2}}(p, k, Q)=\sin \frac{\theta_{f_{1}}(p)-\theta_{f_{1}}(k)}{2} \sin \frac{\theta_{f_{2}}(Q-p)-\theta_{f_{2}}(Q-k)}{2}
\end{gathered}
$$
}

where $f_{1}$ and $f_{2}$ stand for different quark flavours. 
wave function dies out, and the system (2.40) reduces to a single equation for $\varphi_{+}$,

$$
\begin{gathered}
M^{2} \varphi(x)=\left(\frac{m^{2}-2 \gamma}{x}+\frac{m^{2}-2 \gamma}{1-x}\right) \varphi(x)-2 \gamma f \frac{d y}{(y-x)^{2}} \varphi(y), \\
\varphi(x)=\lim _{Q \rightarrow \infty} \sqrt{\frac{Q}{2 \pi}} \varphi_{+}(p, Q),
\end{gathered}
$$

coinciding with the one derived by 't Hooft in [四].

For further references we give here a couple of properties of the wave functions $\varphi_{ \pm}^{n}$ :

$$
\begin{gathered}
\varphi_{ \pm}^{n}(p \mp P, \mp P)=\varphi_{ \pm}^{n}(p, \pm P), \\
\varphi_{ \pm}^{n}(P-p, P)=\eta_{n} \varphi_{ \pm}^{n}(p, P),
\end{gathered}
$$

and the parity of the state is $(-1)^{\eta_{n}+1}$. The latter property allows one to classify all mesonic states into two groups: odd states, pions, with $\eta_{n}=1$, and even states, $\sigma$ 's, with $\eta_{n}=-1$. The odd and the even states follow one by one in the spectrum starting from the lowest state, which is odd and it is expected to be massless in the chiral limit. This state is nothing but the celebrated chiral pion.

\subsection{Pionic solution}

In this subsection we return to the bound-state equation (2.40) and find an exact solution to it. Indeed, one can easily check that the set of the following two functions:

$$
\begin{gathered}
\varphi_{ \pm}^{\pi}(p, Q)=N_{\pi}^{-1}\left(\cos \frac{\theta(Q-p)-\theta(p)}{2} \pm \sin \frac{\theta(Q-p)+\theta(p)}{2}\right) \\
N_{\pi}^{2}(Q)=\frac{2}{\pi} Q
\end{gathered}
$$

satisfies the system (2.40) if the quark mass is put to zero. This solution turns out to be massless and thus it is nothing but the chiral pion - the lowest negative-parity state in the spectrum (see the discussion at the end of the previous subsection). As one should anticipate, the norm of this state (2.46) vanishes in the pion rest frame, whereas in the infinite momentum frame this solution reads

$$
\varphi_{-}^{\pi}(p, Q) \underset{Q \rightarrow \infty}{\rightarrow} 0, \quad \varphi_{+}^{\pi}(p, Q) \underset{Q \rightarrow \infty}{\rightarrow} \sqrt{\frac{2 \pi}{Q}}, \quad 0 \leq p \leq Q
$$

and, after an appropriate rescaling (see equation (2.42), gives $\varphi(x)=1$, i.e., turns into the pionic solution found in [1, 3].

\subsection{The one-particle limit and the nonpotential quark dynamics}

Let us consider a heavy-light system containing a static antiquark source placed at the origin and a light quark. The most straightforward way to derive the bound-state equation for this system is to take the one-body limit of the system (2.40). For the infinitely heavy flavour one has (see the footnote on the page 15)

$$
E_{f_{2}}(p)=m_{f_{2}} \rightarrow \infty, \quad \cos \theta_{f_{2}}=1, \quad \sin \theta_{f_{2}}=0, \quad \varphi_{-}^{f_{1} f_{2}}=0,
$$


then the coefficients in $(2.40)$ become:

$$
C_{f_{1} f_{2}}(p, k, Q)=\cos \frac{\theta_{f_{1}}(p)-\theta_{f_{1}}(k)}{2}, \quad S_{f_{1} f_{2}}(p, k, Q)=0 .
$$

From now on, in this subsection, we shall suppress all flavour indices, having in mind that the angle $\theta(p)$ and the dispersive law $E(p)$ are for the light quark.

The system (2.40) reduces to a single equation [28],

$$
\varepsilon \varphi(p)=E(p) \varphi(p)-\gamma f \frac{d k}{(p-k)^{2}} \cos \frac{\theta(p)-\theta(k)}{2} \varphi(k),
$$

where $\varphi=\varphi_{+}, \varepsilon=Q_{0}-m_{f_{2}}$.

Note that the interaction in (2.50), given by the integral term on the r.h.s., is essentially nonlocal and, moreover, there is no parameter (except the mass of the quark) which could allow one to treat this nonlocality perturbatively, expanding the cosine under the integral. Meanwhile, if the quark is also heavy, then the cosine reduces to unity and equation (2.50) can be simplified even more, yielding the local linearly rising potential between the constituents,

$$
\left(E_{0}(p)+\gamma|x|\right) \varphi(x)=\varepsilon \varphi(x), \quad E_{0}(p)=\sqrt{p^{2}+m^{2}} \approx m+\frac{p^{2}}{2 m}+\ldots
$$

To get a deeper insight into the structure of the interaction in the 't Hooft model let us use another approach to the heavy-light system.

\subsection{A heavy-light system in the modified Fock-Schwinger gauge and the Lorentz nature of confinement}

In this subsection we discuss another way to derive the one-particle limit (2.50) of the boundstate equation (2.40), based on the Schwinger-Dyson equation for $\mathrm{QCD}_{2}$ in the modified Fock-Schwinger gauge (Balitsky gauge) 28]. The advantage of this approach is a possibility of its generalization to $\mathrm{QCD}_{4}$ if an appropriate model for the QCD vacuum is used [17].

First of all, note that the Coulomb gauge condition, $A_{1}\left(x_{0}, x\right)=0$, does not fix the gauge completely allowing purely time-dependent gauge transformations. To fix the residual invariance we impose an extra condition, $A_{0}\left(x_{0}, x=0\right)=0$, 0 which obviously breaks translational invariance, but turns out very convenient if an infinitely heavy (static) particle is involved. In case of the system containing a light quark and a static antiquark source the origin can be associated with the latter, which appears to play an extremely passive role in the interaction providing the white colour of the entire object. The Green's function of the static antiquark is independent of the gluonic field in this gauge, $S_{\bar{Q}}(x, y \mid A)=S_{\bar{Q}}(x-y)$, and takes the form:

$$
S_{\bar{Q}}(x)=\hat{1}(-i)\left(\frac{1+\gamma_{0}}{2} \theta\left(-x_{0}\right) e^{i M x_{0}}+\frac{1-\gamma_{0}}{2} \theta\left(x_{0}\right) e^{-i M x_{0}}\right) \delta(x),
$$

where $\hat{1}$ denotes unity in the colour space. The Green's function (2.52) contains also an infinite set of corrections of the form $\frac{1}{M^{n}} \frac{d^{n}}{d x^{n}} \delta(x-y)$ which die out in the limit $M \rightarrow \infty$.

\footnotetext{
${ }^{6}$ In $3+1$ this gauge is usually introduced via conditions $A_{0}^{a}\left(x_{0}, \overrightarrow{0}\right)=0$ and $\vec{x} \overrightarrow{A^{a}}\left(x_{0}, \vec{x}\right)=0$ [29].
} 
The Green's function of the colourless $q \bar{Q}$ system can be written in the following form:

$$
\begin{aligned}
& S_{q \bar{Q}}(x, y)=\frac{1}{N_{C}} \int D \psi D \bar{\psi} D A_{\mu} \exp \left\{-\frac{i}{4} \int d^{2} x F_{\mu \nu}^{a 2}+i \int d^{2} x \bar{\psi}(i \hat{\partial}-m-\hat{A}) \psi\right\} \times \\
& \times \bar{\psi}(x) S_{\bar{Q}}(x-y) \psi(y),
\end{aligned}
$$

so that it turns out possible to integrate out the gluonic field arriving at a Schwinger-Dyson equation for the light-quark Green's function $S(x, y)$,

$$
\left(i \hat{\partial}_{x}-m\right) S(x, y)-(2 \pi)^{2} \gamma \int d^{2} z \gamma_{0} S(x, z) \gamma_{0} \tilde{D}_{00}(x, z) S(z, y)=\delta^{(2)}(x-y) .
$$

Note that $S(x, y)=\frac{1}{N_{C}} S_{\alpha}^{\alpha}(x, y)$ possesses all properties of the full $q \bar{Q}$ Green's function due to the passive role of the antiquark discussed above. Then both, one-particle (e.g., the chiral condensate), as well as two-particle (e.g., the spectrum of bound states) properties of the system can be extracted from the single function $S(x, y)$. A special attention is to be payed to the gluonic propagator $\tilde{D}_{00}(x, y)$, which looks similarly to that in the Coulomb gauge (11.5) but contains extra terms breaking the translational invariance and encoding the light-quark interaction with the static antiquark,

$$
\tilde{D}_{00}\left(x_{0}-y_{0}, x, y\right)=-\frac{i}{2}(|x-y|-|x|-|y|) \delta\left(x_{0}-y_{0}\right) \equiv K(x, y) \delta\left(x_{0}-y_{0}\right),
$$

or in the momentum space,

$$
\begin{gathered}
K(p, q)=K^{(1)}(p, q)+K^{(2)}(p, q), \\
K^{(1)}(p, q)=\frac{i}{p^{2}} \delta(p-q), \\
K^{(2)}(p, q)=-\frac{i}{q^{2}} \delta(p)-\frac{i}{p^{2}} \delta(q),
\end{gathered}
$$

where we have separated the local and the nonlocal parts.

From now on two different strategies can be adopted, which finally lead to the same equation for the spectrum of the heavy-light system. The first approach is based on a diagrammatic technique with two different internal lines prescribed to the local and the nonlocal parts of the kernel [28. It turns out that, in spite of the nonlinearity of the equation (2.54), the two parts of the kernel can be considered separately due to very peculiar properties of rainbow diagrams 28 . Thus the local part $K^{(1)}$ defines the mass operator $\Sigma(p)$, which can be naturally parametrized by means of the dressed quark dispersive law $E(p)$ and the chiral angle $\theta(p)$,

$$
\Sigma(p)=[E(p) \cos \theta(p)-m]+\gamma_{1}[E(p) \sin \theta(p)-p],
$$

with the system of coupled equations (2.12) being the selfconsistency condition of such a parametrization. Then the nonlocal part $K^{(2)}$ eventually gives the bound-state equation (see [28] for the details).

Here we choose the other strategy based on the Foldy-Wouthoysen transformation of equation (2.54) [30]. First, we rewrite this equation in the momentum space and use a spectral decomposition for the light-quark Green's function,

$$
S\left(q_{10}, q_{1}, q_{20}, q_{2}\right)=2 \pi \delta\left(q_{10}-q_{20}\right)\left(\sum_{\varepsilon_{n}>0} \frac{\varphi_{n}^{(+)}\left(q_{1}\right) \bar{\varphi}_{n}^{(+)}\left(q_{2}\right)}{q_{10}-\varepsilon_{n}+i 0}+\sum_{\varepsilon_{n}<0} \frac{\varphi_{n}^{(-)}\left(q_{1}\right) \bar{\varphi}_{n}^{(-)}\left(q_{2}\right)}{q_{10}+\varepsilon_{n}-i 0}\right)
$$


where the positive- and the negative-energy solutions $\varphi_{n}^{( \pm)}$have been introduced. To proceed further we assume that a Foldy-Wouthoysen operator, $T_{F}(p)=e^{-\frac{1}{2} \theta_{F}(p) \gamma_{1}}$, diagonalizing equation (2.54), exists and that the angle $\theta_{F}$ is the same for all $n$ 's. With such an assumption one has

$$
\begin{gathered}
\varphi_{n}^{(+)}(p)=\varphi_{n}^{0}(p) T_{F}(p)\left(\begin{array}{l}
1 \\
0
\end{array}\right), \quad \varphi_{n}^{(-)}(p)=\varphi_{n}^{0}(p) T_{F}(p)\left(\begin{array}{l}
0 \\
1
\end{array}\right), \\
\int \frac{d p}{2 \pi} \varphi_{n}^{0}(p) \varphi_{m}^{0}(p)=\delta_{n m}, \quad \sum_{n} \varphi_{n}^{0}(p) \varphi_{n}^{0}(q)=2 \pi \delta(p-q),
\end{gathered}
$$

so that the following relation holds true for the Green's function (2.59):

$$
\int \frac{d \omega}{2 \pi} S\left(\omega, q_{1}, q_{2}\right)=-i \pi \delta\left(q_{1}-q_{2}\right)\left[\cos \theta_{F}\left(q_{1}\right)-\gamma_{1} \sin \theta_{F}\left(q_{1}\right)\right]
$$

where $\omega=q_{10}-q_{20}$.

The Schwinger-Dyson equation (2.54) reduces then to a Dirac-type equation in the Hamiltonian form,

$$
(\alpha p+\beta m) \varphi_{n}^{0}(p)-\frac{i \gamma}{2} \int d q d k\left(\beta \cos \theta_{F}(q)+\alpha \sin \theta_{F}(q)\right) K(p-q, k-q) \varphi_{n}^{0}(k)=\varepsilon_{n} \varphi_{n}^{0}
$$

The local part of the interaction in (2.63), generated by $K^{(1)}$, leads to dressing of the light quark described by the Bogoliubov-Valatin angle $\theta(p)$ and the dressed quark dispersive law $E(p)$ obeying the system (2.12). Therefore, one comes to the conclusion that the Foldy angle $\theta_{F}(p)$ coincides with the Bogoliubov-Valatin one,

$$
\theta_{F}(p)=\theta(p)
$$

The nonlocal part of the interaction in (2.63), which stems from $K^{(2)}$, is also diagonalized then, so that one ends with a Schrödinger-type equation,

$$
\varepsilon_{n} \varphi_{n}^{0}(p)=E(p) \varphi_{n}^{0}(p)-\gamma f \frac{d k}{(p-k)^{2}} \cos \frac{\theta(p)-\theta(k)}{2} \varphi_{n}^{0}(k),
$$

which coincides with (2.50).

Comparing bound-state equation (2.65) with (2.40) one finds that $\varphi^{0}$ plays the role of the $\varphi_{+}$component of the heavy-light system wave functions, whereas $\varphi_{-}$vanishes due to presence of the infinitely massive antiquark. Thus relations (2.61) follow immediately from (2.36) and (2.37) with all $\varphi_{-}$'s put to zero.

It is instructive to note that in the Coulomb (as well as Balitsky) gauge the 't Hoof model is totally defined by only one nontrivial function $\theta(p)$, solution to the gap equation (2.13), which plays a threefold role:

- it defines the Bogoliubov-Valatin transformation from bare to dressed quarks;

- it gives the Foldy angle, which diagonalizes the interquark interaction in the model;

- it entirely defines all quantities in the model, including the bound-state equation. 
Several comments concerning equation (2.54) are in order here. The first one deals with the generalization of (2.54) to the four-dimensional case. The attentive reader may notice that the only two-dimensional constituent of (2.54) is the gluonic propagator $\tilde{D}_{\mu \nu}(x, z)$ taken in the form (2.55). The equation itself survives in the case of $\mathrm{QCD}_{4}$, if one has an appropriate form of the bilocal gluonic correlator $\tilde{D}_{\mu \nu}(x, z)$ in the given gauge, and some arguments exist, why higher orders correlators, which lead to many-fermion vertices higher than four, can be neglected (see 31] for details).

Another interesting issue concerning equation (2.54) is that one cannot simplify the interaction kernel substituting

$$
\gamma_{0} S \gamma_{0} \rightarrow \gamma_{0} S_{0} \gamma_{0}
$$

as proposed in [32]. The reason for this failure is discussed in detail in [33] and comes from the fact that the real parameter defining the substitution (2.66), with the consequent expanding of $S_{0}$ in powers of the one-dimensional momentum, is the product of the quark mass and the gluonic correlation length. The latter parameter defines also the radius of the string formed between the colour constituents in the theory. A simple dimensional analysis demonstrates that strings are infinitely thin in $1+1$, as the system has too low dimension to allow them to swell. One can arrive at the same conclusion inspecting the two-dimensional correlator $\langle F F\rangle \equiv \operatorname{Tr}\left\langle F_{\mu \nu}(x) \Phi(x, y) F_{\rho \sigma}(y) \Phi(y, x)\right\rangle$, where $\Phi(x, y)$ is the standard parallel transporter along an arbitrary path between the points $x$ and $y$, which provides the gauge invariance of the entire nonlocal object [21] (see also the review paper [22] for the detailed description of the formalism). Using the gluonic propagator (1.5), one easily finds that $\langle F F\rangle$ is proportional to the two-dimensional $\delta$-function in the configuration space,

$$
\langle F F\rangle \sim \delta^{(2)}(x-y)
$$

i.e., it has zero correlation length $T_{g}$. Thus the product $m T_{g}$ is identically zero in twodimensional QCD, which makes the interaction essentially nonlocal and the quark dynamics becomes not potential [33, 30].

Finally, equation (2.63) answers, at least in $\mathrm{QCD}_{2}$, the long-standing question on the Lorentz nature of confinement. One should distinguish between the Lorentz structure of the confining interaction which is of the $\gamma_{0} \times \gamma_{0}$ type (see (2.54)) and the effective interaction, which enters the Dirac-like equation (2.63), describing the bound-state problem. As clearly seen from (2.63), the latter contains only effective scalar (terms $\sim \gamma_{0}$ ) and space vector (terms $\left.\sim \gamma_{0} \gamma_{1}=\gamma_{5}\right)$ interactions.

\subsection{The chiral properties of the model in the Hamiltonian ap- proach}

In this subsection we discuss the chiral properties of the model which are highly nontrivial and in many features resemble those of four-dimensional QCD.

Most studies of the 't Hooft model have been performed in the light-cone gauge, $A_{-}=$ 0 , which leads to a perturbative vacuum and to a simpler bound-state equation. In the meantime, one has to employ rather sophisticated methods to discuss the chiral limit of the model, when all functions and distributions become extremely singular, and the whole range of the 't Hooft variable definition, $0<x<1$, is squeezed to small intervals near the boundary points $x=0$ and $x=1$, which deliver all nontrivial content of the theory. The Hamiltonian approach in the Coulomb gauge developed above is free of this drawback. Indeed, to calculate 
a matrix element of any operator between mesonic states, the only relevant ones in the weak regime of the model, one is to rewrite the above-mentioned operator in terms of operators $\mathrm{m}^{+}$ and $m$ introduced in (2.34) and to use the second quantization technique to evaluate directly the matrix element. The result is always expressed in terms of trigonometric functions of the angle $\theta(p)$ and integrals of them, which can be worked out analytically in some cases, or treated numerically. Anyway, with the numerical solution for $\theta$ found in [5] any value in the model appears calculable.

Let us make just one more comment concerning the vacuum of the theory. As it was mentioned above, the true vacuum of the model is the mesonic one annihilated by the mesonic operators,

$$
m_{n}(P)|\Omega\rangle=0
$$

for any $n$ and any total momentum $P$. By a unitary transformation this state is related to the quark vacuum defined by relations (2.3),

$$
|\Omega\rangle=U|0\rangle .
$$

Despite of the fact that the explicit form of the operator $U$ is unknown, the difference between the averages calculated with the help of the mesonic and the quark vacua turns out to be suppressed in the large- $N_{C}$ limit, so that it plays the role of a small correction and, hence, lies beyond the scope of the present paper. Thus for practical calculations one is free to use any of the above two vacua.

\subsubsection{The chiral condensate}

A crucial test for the chiral symmetry, to see if it is spontaneously broken or not, is the chiral condensate $\langle\bar{q} q\rangle$. If this average does not vanish for the vacuum state, then the whole tower of physical states will lack the chiral symmetry respected by the Hamiltonian of the theory, so that this symmetry appears spontaneously broken. For the simplest case of only one quark flavour the chiral symmetry breaking reads $U(1)_{L} \times U(1)_{R} \rightarrow U(1)_{V}$, and it is very important that the $U(1)_{A}$ invariance is broken spontaneously in the 't Hooft model, in contrast to $\mathrm{QCD}_{4}$ where this breaking is explicit due to the axial anomaly. Indeed, the two-dimensional anomaly is proportional to the colour trace of the coloured object $\tilde{F} \sim$ $\varepsilon_{\mu \nu} F_{\mu \nu}$, which obviously vanishes (in the meantime, the axial anomaly does exist in the two-dimensional QED, known as the Schwinger model, where no colour trace should be taken).

Now we are in the position to evaluate the chiral condensate for the 't Hooft model. Following the general approach described at the beginning of this subsection, one can find

$$
\begin{aligned}
& \langle\bar{q} q\rangle=\left\langle\Omega\left|\bar{q}_{\alpha}(x) q^{\alpha}(x)\right| \Omega\right\rangle \underset{N_{C} \rightarrow \infty}{\approx}\left\langle 0\left|\bar{q}_{\alpha}(x) q^{\alpha}(x)\right| 0\right\rangle= \\
& =N_{C} \int \frac{d k}{2 \pi} \operatorname{Tr}\left\{\gamma_{0} \Lambda_{-}(k)\right\}=-\frac{N_{C}}{\pi} \int_{0}^{+\infty} d k \cos \theta(k) .
\end{aligned}
$$

It is instructive to arrive at the same result using another approach - namely, the definition of the condensate via the light-quark Green's function. In spite of the fact that the single quark Green's function is a gauge variant object and, hence, it is not physical by 
itself, when taken with the coinciding arguments and summed up over the colours, it readily gives the chiral condensated

$$
\langle\bar{q} q\rangle=-i \underset{x \rightarrow y+}{\operatorname{Tr}} S_{\alpha}^{\alpha}(x, y) .
$$

Once the relation (2.71) is gauge invariant, then let us choose the modified Fock-Schwinger gauge discussed above. The quark Green's function, $S(x, y)=\frac{1}{N_{C}} S_{\alpha}^{\alpha}(x, y)$, is the solution to equation (2.54) [28]. Using its spectral decomposition in the coordinate space,

$$
\begin{gathered}
S\left(x_{0}-y_{0}, x, y\right)=-i \sum_{n} \psi_{n}^{(+)}(x) \bar{\psi}_{n}^{(+)}(y) e^{-i \varepsilon_{n}\left(x_{0}-y_{0}\right)} \theta\left(x_{0}-y_{0}\right)+ \\
+i \sum_{n} \psi_{n}^{(-)}(x) \bar{\psi}_{n}^{(-)}(y) e^{i \varepsilon_{n}\left(x_{0}-y_{0}\right)} \theta\left(y_{0}-x_{0}\right) \\
\psi_{n}^{( \pm)}(x)=\int_{-\infty}^{\infty} \frac{d p}{2 \pi} \varphi_{n}^{( \pm)}(p) e^{i p x}
\end{gathered}
$$

where $\varepsilon_{n}$ are the eigenenergies defined by equation (2.65), substituting the Foldy-rotated wave functions (2.60),

$$
\begin{aligned}
& \varphi_{n}^{(+)}(p)=T^{+}(p) \tilde{\varphi}_{n}^{(+)}(p)=\varphi_{n}^{0}(p) T^{+}(p)\left(\begin{array}{l}
1 \\
0
\end{array}\right), \\
& \varphi_{n}^{(-)}(p)=T^{+}(p) \tilde{\varphi}_{n}^{(-)}(p)=\varphi_{n}^{0}(p) T^{+}(p)\left(\begin{array}{l}
0 \\
1
\end{array}\right),
\end{aligned}
$$

and, finally, using the simplified completeness condition (2.61) for the set $\left\{\varphi_{n}^{0}(p)\right\}$, one reproduces the result (2.70) 28].

It is easily seen from the definition (2.71) that diagrammatically the chiral condensate can be represented as a closed fermion line which begins and ends at the coinciding points. Such way both, the motion forward in time (the positive-energy solutions) and the one backward in time (the negative-energy solutions), are equally important for the condensate. At first glance this statement contradicts the observation made above, that all $\varphi_{-}$components vanish for the heavy-light system. Solution of this problem can be found in properties of the boundstate equation (2.40). Indeed, there are, in fact, two sets of solutions to the system (2.40), with $Q_{0}>0$ and $Q_{0}<0$, trivially connected with one another,

$$
\varphi_{+}^{-n}(p, P)=\varphi_{-}^{n}(p, P), \quad \varphi_{-}^{-n}(p, P)=\varphi_{+}^{n}(p, P)
$$

where positive $n$ 's numerate states with $Q_{0}>0$, and negative $n$ 's are prescribed to the states with $Q_{0}<0$. Therefore, these are $\varphi_{+}^{-n}(p, P)=\varphi_{-}^{n}(p, P)(n>0)$ to vanish for the heavylight system, whereas the two remaining wave functions $\varphi_{-}^{-n}(p, P)=\varphi_{+}^{n}(p, P)$ describe the propagation of the $q \bar{Q}$ system either forward or backward in time without Zitterbewegung. They both contribute on equal footing to the chiral condensate (2.71).

From (2.70) one can see that the properties of the solution for $\theta$ to the gap equation (2.13) are of paramount importance for the chiral symmetry breaking. If the integral on the r.h.s. in (2.70) vanishes, then we are in the phase of the theory with the restored chiral symmetry.

\footnotetext{
${ }^{7} \mathrm{~A}$ special care should be taken at the both stages. Indeed, the Green's function contains a discontinuity at $x=y$, so that one should approach this limit either from the side of larger, or smaller $y$ 's. On the other hand, if the condensate is calculated beyond the chiral limit, then the logarithmically-ultraviolet-divergent perturbative contribution, proportional to the quark mass, should be subtracted from (2.71).
} 
It was mentioned in subsection 2.1 that there exist two different solutions to the gap equation (2.13) in the chiral limit. One of them, found analytically in [2], gives $|\theta(p)|=\pi / 2$ and, hence, $\cos \theta(p)=0$ everywhere, so that the chiral condensate (2.70) vanishes for this solution (as was discussed above, this phase has an infinite energy and, hence, never realises). Luckily it is not so for the numerical solution found in [5] and depicted in Fig.2. Substituting it into (2.70) and working out the integral numerically one finds:

$$
\langle\bar{q} q\rangle_{m=0}=-0.29 N_{C} \sqrt{2 \gamma}
$$

that coincides with the results found in [6] The chiral symmetry is spontaneously broken in this phase of the theory and the pion, found in the subsection 2.5, is, indeed, the corresponding Goldstone boson. Note that the chiral condensate for the 't Hooft model is known analytically for any value of the quark mass [7].

Now we can return to formula (2.23) for the vacuum energy and to rewrite it using a more physically transparent language. Indeed, applying the same transformation, $\theta(p) \rightarrow \theta(p / A)$, to the chiral condensate (2.70), one easily finds that $\Sigma \equiv\langle\bar{q} q\rangle$ scales linearly with $A$,

$$
\Sigma \rightarrow A \Sigma
$$

so that the mute parameter $A$ can be changed for the chiral condensate and relation (2.23) can be written as

$$
\Delta \mathcal{E}_{v}=C_{1}^{\prime}\left[\frac{1}{2}\left(\frac{\Sigma}{\Sigma_{0}}\right)^{2}-\ln \left|\frac{\Sigma}{\Sigma_{0}}\right|\right]+\gamma C_{3}^{\prime},
$$

where the minimum of the vacuum energy is reached for $\Sigma=\Sigma_{0}$ given by (2.76). If the quark mass does not vanish, then the vacuum energy density (2.78) acquires an extra contribution,

$$
\Delta \mathcal{E}_{v} \rightarrow \Delta \mathcal{E}_{v}+m\left(\frac{\Sigma_{0}}{N_{C}}\right)\left(\frac{\Sigma}{\Sigma_{0}}\right)
$$

which explicitly breaks the invariance of $\Delta \mathcal{E}_{v}$ with respect to the change $\Sigma \rightarrow-\Sigma$. Then the lowest (pionic) excitation over the vacuum with the wrong sign of the condensate acquires an imaginary mass and becomes the tachyon as it follows immediately from the Gell-MannOakes-Renner relation (see equation (3.20)).

Minimization procedure for the vacuum energy in presence of the mass term (2.79) leads to a more complicated equation for $\Sigma$, so that $\Sigma_{0}$ does not provide the minimum anymore.

\subsubsection{The pion decay constant}

In this subsection we derive the decay constant $f_{\pi}$ for the chiral pion. Using the standard definition for it,

$$
\left\langle\Omega\left|J_{\mu}^{5}(x)\right| \pi(Q)\right\rangle=f_{\pi} Q_{\mu} \frac{e^{-i Q x}}{\sqrt{2 Q_{0}}},
$$

${ }^{8}$ The corresponding result from [6] reads

$$
\langle\bar{q} q\rangle_{m=0}=-\frac{N_{C} \sqrt{\gamma}}{\sqrt{6}}
$$

that numerically coincides with (2.76), thus giving evidence that various integrals of $\theta$ can be found not only numerically, but also in the form of irrational numbers. 
where

$$
J_{\mu}^{5}(x)=\bar{q}(x) \gamma_{\mu} \gamma_{5} q(x),
$$

one can calculate the matrix element explicitly using the technique described above, so that the result reads

$$
f_{\pi}=\sqrt{\frac{N_{C}}{\pi}} .
$$

Note that the pion decay constant (2.82) is dimensionless in the 't Hooft model, which drastically differs in this point from $\mathrm{QCD}_{4}$, where this constant is dimensional and it appears rather small at the hadronic scale $(93 \mathrm{MeV})$. Thus in the four-dimensional case the pion decay constant defines a new scale for the effective low-energy QCD - the chiral perturbation theory, which cannot be developed in the 't Hooft model in view of the dimensionlessness of $f_{\pi}$.

\subsubsection{Partial conservation of the axial-vector current (PCAC)}

In this subsection we derive explicitly the PCAC relation for the 't Hooft model.

Starting from the definition of the axial-vector current (2.81), we use representation (2.2) for the quark fields and introduce bosonic operators (2.25) after an appropriate ordering of the antiquark creation and annihilation operators $d^{+}$and $d$. Leaving only the leading terms in the $1 / N_{C}$ expansion, one arrives at

$$
\begin{gathered}
J_{\mu}^{5}(x)=N_{C} \int \frac{d k}{2 \pi} v^{+}(-k) \gamma_{\mu} \gamma_{5} v(-k)+ \\
\sqrt{N_{C}} \int \frac{d p d P}{(2 \pi)^{2}} e^{-i P x}\left[M^{+}(p, p-P) u^{+}(p-P) \gamma_{\mu} \gamma_{5} v(-p)+M(p-P, p) v^{+}(P-p) \gamma_{\mu} \gamma_{5} u(p)\right] .
\end{gathered}
$$

The explicit form of the quark amplitudes $u$ and $v$ given by (2.5) together with the definition of the mesonic creation and annihilation operators (2.34) allows one to proceed further and to rewrite the components of the axial-vector current in the form

$$
\begin{aligned}
& J_{0}^{5}(x)=2 \sqrt{N_{C}} \int \frac{d P}{2 \pi} N_{\pi} e^{-i P x} \sum_{n=0}^{\infty}\left(m_{n}(P)-m_{n}^{+}(-P)\right) \int \frac{d p}{2 \pi} g_{\pi}(p, P) f_{n}(p, P), \\
& J_{1}^{5}(x)=2 \sqrt{N_{C}} \int \frac{d P}{2 \pi} N_{\pi} e^{-i P x} \sum_{n=0}^{\infty}\left(m_{n}(P)+m_{n}^{+}(-P)\right) \int \frac{d p}{2 \pi} f_{\pi}(p, P) g_{n}(p, P),
\end{aligned}
$$

where

$$
f_{n}(p, P)=\frac{1}{2}\left(\varphi_{+}^{n}(p, P)-\varphi_{-}^{n}(p, P)\right), \quad g_{n}(p, P)=\frac{1}{2}\left(\varphi_{+}^{n}(p, P)+\varphi_{-}^{n}(p, P)\right) .
$$

From (2.36) one easily finds that

$$
\int \frac{d p}{2 \pi} g_{\pi}(p, P) f_{n}(p, P)=\int \frac{d p}{2 \pi} f_{\pi}(p, P) g_{n}(p, P)=\frac{1}{4} \delta_{n \pi},
$$

i.e., in the chiral limit the axial-vector current couples only to pions, and it can be written as

$$
J_{\mu}^{5}(x)=i \sqrt{\frac{N_{C}}{\pi}} \partial_{\mu} \int \frac{d P}{2 \pi} \frac{1}{\sqrt{2 P_{0}}}\left(e^{-i P x} m_{\pi}(P)+e^{i P x} m_{\pi}^{+}(P)\right)=i f_{\pi} \partial_{\mu} \Psi_{\pi}(x),
$$


where the second-quantized wave function of the pion in the coordinate space $\Psi_{\pi}(x)$ is introduced.

Relation (2.88) is nothing but the celebrated partial conservation of the axial-vector current (PCAC), whose operator form is usually formulated as a hypothesis in $\mathrm{QCD}_{4}$. In the 't Hooft model the latter can be proved explicitly and the Hamiltonian approach to the model turns out the most natural environment for this task.

It is instructive to note that the form of the pionic solution can be easily guessed even before the : $H_{4}$ : part of the Hamiltonian is taken into account. Indeed, if the chiral symmetry is spontaneously broken, then the corresponding charge does not commute with the Hamiltonian,

$$
\left[Q_{5} H\right] \neq 0, \quad Q_{5}=\int d x J_{0}^{5}(x),
$$

so that, if the Hamiltonian is diagonalized in the quark sector, then $Q_{5}$ contains an anomalous term,

$$
Q_{5} \sim \int \frac{d p}{2 \pi}\left[b^{+}(p) d^{+}(-p)+d(-p) b(p)\right] \cos \theta(p),
$$

with the coefficient $\cos \theta(p)$ being right the pion wave function in the rest frame (see equations (2.45) and (3.24)).

\section{Matrix approach}

In spite of evident technical advantages and physical transparency of the Hamiltonian approach developed and exploited in the previous section, it has a number of disadvantages. Among those are a rather tedious algebra and not straightforward connection to the diagrammatic technique which is very convenient in studies of variety of hadronic processes. In the present section we develop a matrix approach to the 't Hooft model, which allows one to simplify considerably investigations of some phenomena, e.g., this technique will be effectively used in the next section, where the Ward identities and the strong hadronic decays are discussed.

The section is organized as follows. In subsection 3.1, following [2], we introduce the matrix wave function and derive the bound-state equation for it. In subsection 3.2 we study properties of the matrix Hamiltonian - namely, its Hermiticity and the Hilbert space of its definition. Chiral properties of the 't Hooft model are the subject of the next subsection 3.3. We establish the Gell-Mann-Oakes-Renner relation, discuss the pionic wave function beyond the chiral limit, and return to the calculation of the pion decay constant. In the consequent subsections 3.4, 3.5, and 3.6 we derive the quark-quark scattering amplitude and Ward identities for the vector and axial-vector currents and find the pionic vertex, respectively.

\subsection{Matrix wave functions and matrix bound state equation}

In this subsection we briefly recall the method and the results of the paper [2] based on the diagrammatic approach to the theory.

At the first step we define the mass operator $\Sigma$ as a sum of planar diagrams (see Fig.(1) 


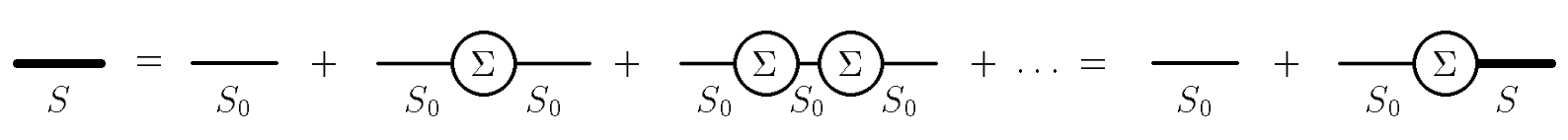

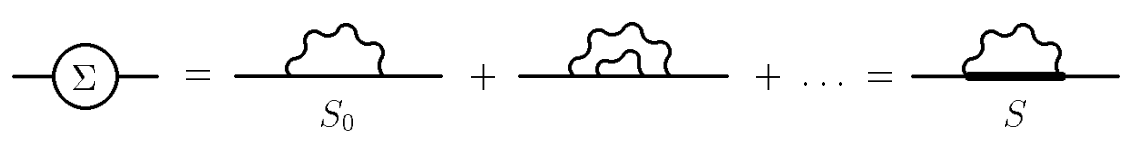

Figure 4: Graphical representation of the equations for the dressed quark propagator and the quark mass operator.

which contribute to the dressed quark propagator $S\left(p_{\mu}\right)$. 9

$$
\begin{gathered}
S\left(p_{\mu}\right)=\frac{1}{\hat{p}-m-\Sigma(p)+i \varepsilon}, \\
\Sigma(p)=\frac{i \gamma}{2 \pi} \int \frac{d k_{0} d k}{(p-k)^{2}} \gamma_{0} S\left(k_{\mu}\right) \gamma_{0} .
\end{gathered}
$$

Note that due to the instantaneous type of the interaction the integration over $k_{0}$ is trivial and the mass operator depends only on the spatial component of the momentum. Using the same parametrization as in (2.58) one immediately arrives at the gap equation in the form (2.13) and the definition of $E(p)$ via $\theta(p)$ coinciding with (2.14).

As the second step a homogeneous Bethe-Salpeter equation is used, which is diagrammatically represented in Fig.5 and defines the spectrum of the quark-antiquark bound-states. The fat lines denote the dressed quark propagators (3.1) whereas the meson-quark-antiquark vertices are described by the function $\Gamma(p, P)$. It is also convenient to introduce a modified vertex $\tilde{\Gamma}\left(p_{\mu}, P_{\mu}\right)$,

$$
\tilde{\Gamma}\left(p_{\mu}, P_{\mu}\right)=-i \gamma S(p) \Gamma(p, P) S(p-P),
$$

and a matrix wave function $\Phi(p, Q)$ defined in the standard way [2],

$$
\Phi(p, Q)=\int \frac{d p_{0}}{2 \pi} \tilde{\Gamma}\left(p_{\mu}, Q_{\mu}\right)=\int \frac{d p_{0}}{2 \pi} \tilde{\Gamma}\left(p_{0}-Q_{0}, p, Q\right)
$$

The equation corresponding to the diagrams in Fig.5 reads

$$
\tilde{\Gamma}\left(p_{\mu}, Q_{\mu}\right)=\frac{i \gamma}{2 \pi} \int \frac{d k_{0} d k}{(p-k)^{2}} S\left(p_{\mu}\right) \gamma_{0} \tilde{\Gamma}\left(k_{\mu}, Q_{\mu}\right) \gamma_{0} S\left(p_{\mu}-Q_{\mu}\right)
$$

or, after integrating both sides of this equation over $p_{0}$, introduction of the wave function $\Phi$ according to relation (3.4), and performing simple algebraic transformations, one arrives at the bound-state equation in the matrix form,

$$
\begin{aligned}
Q_{0} \Phi(p, Q) & =\left(\gamma_{5} p+\gamma_{0} m\right) \Phi(p, Q)-\Phi(p, Q)\left(\gamma_{5}(Q-p)+\gamma_{0} m\right) \\
& +\gamma \int \frac{d k}{(p-k)^{2}}\left\{\Lambda_{+}(k) \Phi(p, Q) \Lambda_{-}(Q-k)-\Lambda_{+}(p) \Phi(k, Q) \Lambda_{-}(Q-p)\right.
\end{aligned}
$$

\footnotetext{
${ }^{9}$ We use the argument $p_{\mu}$ as a shorthand notation for $\left(p_{0}, p\right)$. If not stated explicitly, then dependence only on the one-dimensional spatial momentum is meant, like in $\Sigma(p)$.
} 

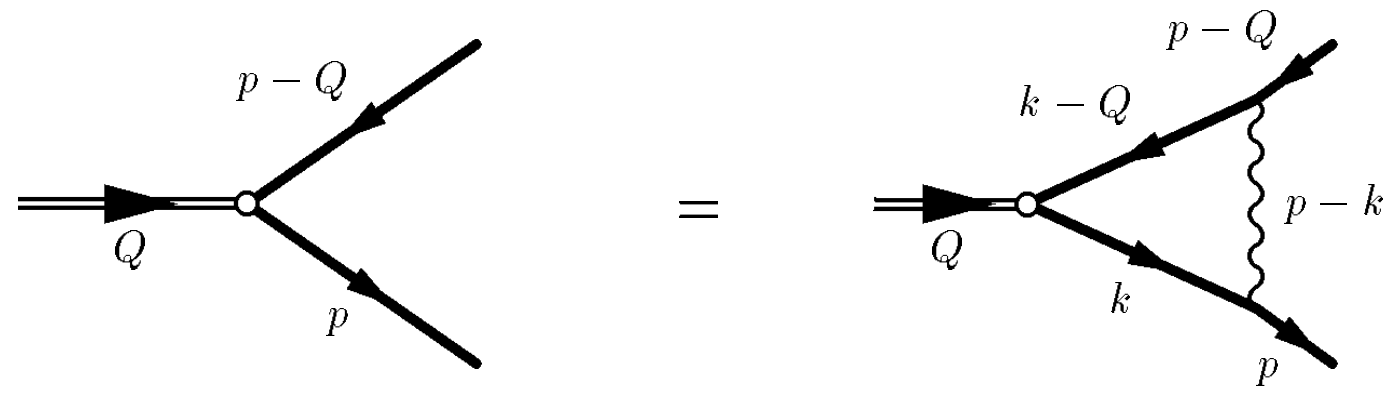

Figure 5: Graphical representation for the Bethe-Salpeter equation (3.5).

$$
\left.-\Lambda_{-}(k) \Phi(p, Q) \Lambda_{+}(Q-k)+\Lambda_{-}(p) \Phi(k, Q) \Lambda_{+}(Q-p)\right\},
$$

where we used projectors (2.11) and the matrix wave function is parametrized as

$$
\Phi(p, Q)=T(p)\left(\frac{1+\gamma_{0}}{2} \gamma_{5} \varphi_{+}(p, Q)+\frac{1-\gamma_{0}}{2} \gamma_{5} \varphi_{-}(p, Q)\right) T^{+}(Q-p) .
$$

When written in components, equation (3.6) readily reproduces the bound-state equation (2.40) for the functions $\varphi_{ \pm}$derived earlier in the framework of the Hamiltonian approach to the model.

In conclusion we give the connection between the meson-quark-antiquark vertex $\Gamma(p, P)$ and the matrix wave function $\Phi(p, P)$ :

$$
\Gamma(p, P)=\int \frac{d k}{2 \pi} \gamma_{0} \frac{\Phi(k, P)}{(p-k)^{2}} \gamma_{0}, \quad \bar{\Gamma}(p, P)=\gamma_{0} \Gamma^{+}(p, P) \gamma_{0},
$$

where the vertices $\Gamma$ and $\bar{\Gamma}$ stand for the incoming and the outgoing mesons, respectively. It is easy to check that, with such a definition, relation (3.4) is satisfied automatically (see also Appendix A for the properties of $\Gamma$ and $\bar{\Gamma}$ ).

\subsection{Truncated Hilbert space and the problem of Hermiticity}

In this subsection we discuss properties of the matrix bound-state equation (3.6), but let us make a comment concerning its scalar form (2.40) first. As stated before, the norm of the wave functions $\varphi_{ \pm}(2.36)$ is defined in an unusual way. Indeed, the sign minus between its "+" and "-" parts appears quite naturally in the Bogoliubov-like approach developed above, but it looks somewhat surprisingly in the context of the standard Hamiltonian technique. Besides, it is easy to check that, if the matrix bound-state equation (3.6) is written in the Schrödinger-like form,

$$
Q_{0}^{n}\left(\begin{array}{c}
\varphi_{+}^{n} \\
\varphi_{-}^{n}
\end{array}\right)=\hat{\mathcal{H}}\left(\begin{array}{c}
\varphi_{+}^{n} \\
\varphi_{-}^{n}
\end{array}\right)
$$

then the corresponding Hamiltonian $\hat{\mathcal{H}}$ appears non-Hermitian. The following two questions should be discussed in this connection: i) what is the reason for this, and ii) whether this does not lead to a disaster and the eigenenergies of this equation are still real.

The answer to the first question becomes clear if one notices that the matrix wave function (3.7) satisfies the following conditions:

$$
\Lambda_{+}(p) \Phi(p, Q) \Lambda_{+}(Q-p)=\Lambda_{-}(p) \Phi(p, Q) \Lambda_{-}(Q-p)=0,
$$


so the phase space is truncated and the Hamiltonian $\hat{\mathcal{H}}$ acts in a subspace, that explains also the distorted norm (2.36).

In the meantime, the second question concerning the spectrum persists. In order to answer it, let us integrate both sides of (3.6) over $p$, do the same for the complex conjugated equation, and take an appropriate linear combination. Then one arrives at the relation

$$
\sum_{n=-\infty}^{+\infty}\left(Q_{0}^{n}-Q_{0}^{m *}\right) \int \frac{d p}{2 \pi}\left(\varphi_{+}^{n}(p, Q) \varphi_{+}^{m}(p, Q)-\varphi_{-}^{n}(p, Q) \varphi_{-}^{m}(p, Q)\right)=0
$$

which immediately leads to the following two conclusions:

$$
Q_{0}^{n}=Q_{0}^{n *}
$$

and

$$
\begin{aligned}
& \int \frac{d p}{2 \pi}\left(\varphi_{+}^{n}(p, Q) \varphi_{+}^{m}(p, Q)-\varphi_{-}^{n}(p, Q) \varphi_{-}^{m}(p, Q)\right)=\delta_{n m} \\
& \int \frac{d p}{2 \pi}\left(\varphi_{+}^{n}(p, Q) \varphi_{-}^{m}(p, Q)-\varphi_{-}^{n}(p, Q) \varphi_{+}^{m}(p, Q)\right)=0 .
\end{aligned}
$$

It was already mentioned before (subsection "Chiral condensate" above) that solutions of the system (2.40) appear in pairs: for each eigenvalue $Q_{0}^{n}$ with the eigenfunction $\left(\varphi_{+}^{n}, \varphi_{-}^{n}\right)$ there exists another eigenvalue, $-Q_{0}^{n}$, with the eigenfunction $\left(\varphi_{-}^{n}, \varphi_{+}^{n}\right)$. With this symmetry, equation (3.13) can be rewritten in the form (2.36) where only positive eigenvalues enter $\square$. Similarly in attempts to construct the Green's function for the system (2.40) the completeness (2.37) can be derived.

Let us introduce operators $\hat{C}$ and $\hat{S}$ :

$$
\hat{C}(\hat{S}) F(p, P) \equiv \gamma \int \frac{d k}{(p-k)^{2}} C(S)(p, k, P) F(k, P)
$$

for an arbitrary function $F(p, P)$ with $C(p, k, P)$ and $S(p, k, P)$ defined in (2.30).

Then the matrix Hamiltonian $\hat{\mathcal{H}}$ can be written in the form

$$
\hat{\mathcal{H}}=\left(\begin{array}{cc}
K-\hat{C} & \hat{S} \\
-\hat{S} & -K+\hat{C}
\end{array}\right)=\gamma_{0}(K-\hat{C})+\gamma_{1} \hat{S},
$$

where $K \equiv E(p)+E(P-p)$ is the kinetic energy. This is the term proportional to $\gamma_{1}$ in (3.15), which makes the Hamiltonian $\hat{\mathcal{H}}$ non-Hermitian. The symmetry property of the solution with respect to interchange of the plus and the minus components of the wave function discussed above follows immediately from the fact that $\hat{\mathcal{H}}$ anticommutes with $\gamma_{5}$, so that if $\psi=\left(\begin{array}{l}\varphi_{+} \\ \varphi_{-}\end{array}\right)$ is the eigenfunction corresponding to the eigenvalue $Q_{0}$, then $\psi^{\prime}=\left(\begin{array}{l}\varphi_{-} \\ \varphi_{+}\end{array}\right)=\gamma_{5} \psi$ is also a solution with the eigenvalue $-Q_{0}$,

$$
\hat{\mathcal{H}} \psi^{\prime}=\hat{\mathcal{H}} \gamma_{5} \psi=-\gamma_{5} \hat{\mathcal{H}} \psi=-\gamma_{5} Q_{0} \psi=-Q_{0} \psi^{\prime} .
$$

Moreover, the eigenstate problem (3.9) for the operator $\hat{\mathcal{H}}$ can be formulated now in the form of an effective Dirac-type equation,

$$
\left[\gamma_{0}(K-\hat{C})+\gamma_{1} \hat{S}-Q_{0}\right] \psi=0
$$

\footnotetext{
${ }^{10}$ If not stated explicitly, we use the symbol $\sum_{n}$ for summation over positive $n$ 's only.
} 
where, as before,

$$
\psi=\left(\begin{array}{c}
\varphi_{+} \\
\varphi_{-}
\end{array}\right)
$$

Mapping of the quark-antiquark bound-states problem to the properties of the fermionictype equation (3.17) may be continued, which is, however, beyond the scope of the present paper.

\subsection{The chiral properties of the model in the matrix approach}

In this subsection we return to the chiral properties of the 't Hooft model and discuss some of them in the framework of the matrix formalism.

In Appendix A we collect formulae useful for various calculations in the suggested approach. They are entirely based on the definition of the matrix wave function (3.7) and properties of the matrix bound-state equation (3.6).

\subsubsection{The Gell-Mann-Oakes-Renner relation and the mass of the pion}

In order to demonstrate how the matrix approach works in practice let us derive the GellMann-Oakes-Renner relation for the 't Hooft model. We slightly relax the chiral limit introducing a small quark mass $m$. The matrix bound-state equation (3.6) is the main object of investigation now. We multiply it by $\gamma_{0} \gamma_{5}$, take trace over spinor indices and integrate both sides of the resulting equation over the momentum $p$. A number of terms containing projectors $\Lambda_{ \pm}$disappears and the result reads

$$
Q_{0} \int \frac{d p}{2 \pi} S p\left[\gamma_{0} \gamma_{5} \Phi(p, Q)\right]-Q \int \frac{d p}{2 \pi} S p\left[\gamma_{1} \gamma_{5} \Phi(p, Q)\right]=-2 m \int \frac{d p}{2 \pi} S p\left[\gamma_{5} \Phi(p, Q)\right] .
$$

If one uses the definition of the matrix wave function and substitutes the pion solution (2.45) into it, then relation (3.19) simplifies even more and takes the form:

$$
f_{\pi}^{2} M_{\pi}^{2}=-2 m\langle\bar{q} q\rangle
$$

in which one can easily recognize the celebrated Gell-Mann-Oakes-Renner relation 34. This defines the mass of the pion near the chiral limit,

$$
M_{\pi}^{2}=2 m \int_{0}^{\infty} d p \cos \theta(p)
$$

With the help of the numerical solution for $\theta$ (see Fig.2) and the footnote at page 23 one can find:

$$
M_{\pi}^{2}=\sqrt{\frac{2 \pi^{2} m^{2} \gamma}{3}} \sim m \sqrt{\gamma} .
$$

Note that in the case of the chirally invariant vacuum, i.e., for the analytic solution (2.18), equation (3.20) is trivial as its both sides vanish simultaneously. 

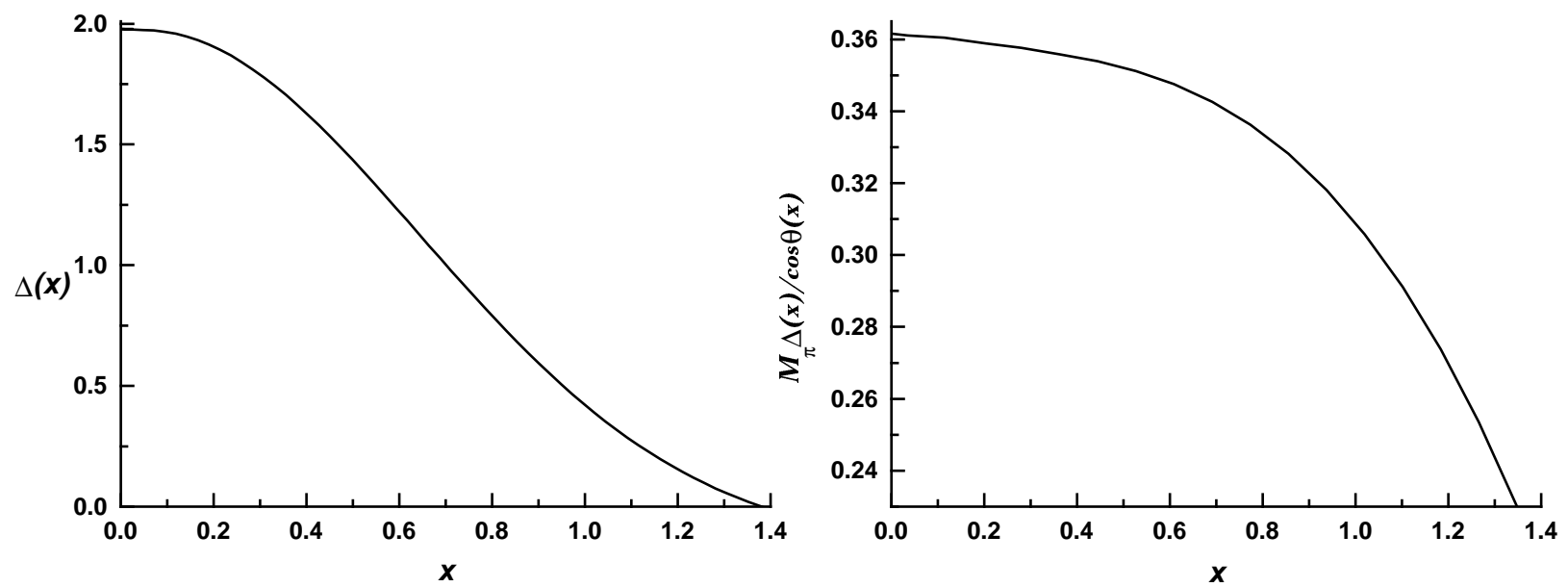

Figure 6: Numerical solution for the function $\Delta$ and the ratio of the subleading term in (3.24) to the leading one for $m=0.18$. As before, variable $x$ comes from the change $p=\tan (x)$, all dimensional quantities are given in proper units of $(2 \gamma)^{1 / 2}$.

\subsubsection{The pionic solution beyond the chiral limit}

With the pion mass (3.22) in hands we are in the position to go slightly beyond the chiral limit and to study the pionic solution in the rest frame. A simple analysis demonstrates that in the pion rest frame the functions $g_{\pi}(p, P)$ and $f_{\pi}(p, P)$ defined in (2.86) behave like

$$
g_{\pi}(p, P=0) \sim \frac{1}{\sqrt{M_{\pi}}} \cos \theta(p)+O\left(M_{\pi}^{3 / 2}\right), \quad f_{\pi}(p, P=0) \sim O\left(\sqrt{M_{\pi}}\right) .
$$

Therefore, the leading correction to the pion wave function comes from $f_{\pi}(p, P)$ and $\varphi_{ \pm}^{\pi}(p, P=0)$ can be parametrized in the form (see (2.45)):

$$
\varphi_{ \pm}^{\pi}(p, P=0)=\tilde{N}_{\pi}^{-1}\left[\frac{\cos \theta(p)}{\sqrt{M_{\pi}}} \pm \sqrt{M_{\pi}} \Delta(p)\right],
$$

where we have extracted the explicit dependence of the coefficients on the small pion mass, so that the unknown correction function $\Delta(p)$ (note that $\Delta(p)$ has the same parity as $\cos \theta(p)$, i.e., it is even) does not depend on $M_{\pi}$. The dimensionless norm $\tilde{N}_{\pi}$ is

$$
\tilde{N}_{\pi}^{2}=4 \int \frac{d p}{2 \pi} \Delta(p) \cos \theta(p),
$$

providing the correct normalization for $\varphi_{ \pm}^{\pi}$.

Substituting (3.24) into (2.40), one arrives at a system of two equations, the first of which is satisfied identically due to (2.12), whereas the other one defines the correction function $\Delta(p)$,

$$
\frac{1}{2} \cos \theta(p)-p \Delta(p) \sin \theta(p)=\frac{\gamma}{2} f \frac{d k}{(p-k)^{2}}[\Delta(p)-\Delta(k)] \cos [\theta(p)-\theta(k)] .
$$

Equation (3.26) is subject to numerical studies which are beyond the scope of the present paper. 

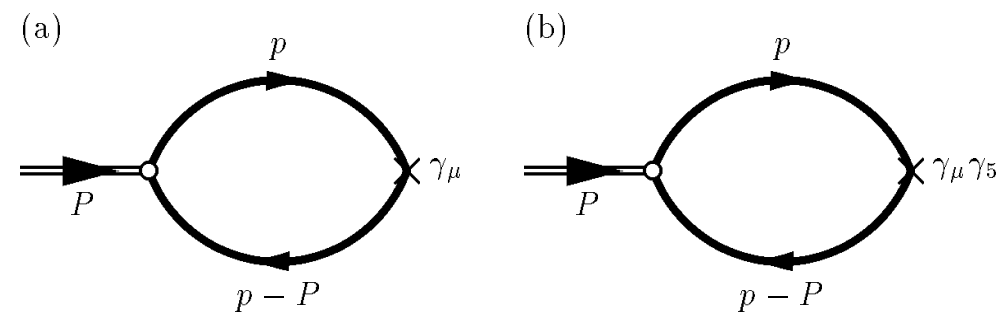

Figure 7: The meson-vector (figure (a)) and meson-axial-vector (figure (b)) current couplings.

Exact numerical solutions for the pion wave functions in the rest frame for several values of the quark mass taken from [5] are given in Fig.3, so that the function $\Delta(p)$ can be extracted directly from these data. In Fig. 6 we give the form of the function $\Delta(p)$ and the ratio of the correction defined by $\Delta(p)$ to the leading term in (3.24) for $m=0.18$ (in units of $\sqrt{2 \gamma}$ ). One can see from the right plot in Fig.6 that for the given value of the quark mass the correction is of order one third at largest and decreases with the growth of the argument.

\subsubsection{The pion decay constant revisited}

In this subsection we give another example of calculations using the matrix approach namely, we calculate the pion decay constant once again, which comes now from the fish-like diagram depicted in Fig.7(b).

The matrix element written for this diagram reads

$$
A_{\mu}=\frac{-i \gamma}{\sqrt{N_{C}}} N_{C}(-1) \int \frac{d^{2} p}{(2 \pi)^{2}} S p\left[S(p) \Gamma_{n}(p, P) S(p-P) \gamma_{\mu} \gamma_{5}\right],
$$

where the factor $-i \gamma / \sqrt{N_{C}}$ comes from the vertex, whereas $N_{C}$ and $(-1)$ are due to the fermionic loop. Working out the integral over $p_{0}$ and using relations (A.7) and (A.8), one arrives at a simple formula,

$$
A_{\mu}=\sqrt{N_{C}} \int \frac{d p}{2 \pi} S p\left[\Phi_{n}(p, P) \gamma_{\mu} \gamma_{5}\right] .
$$

Then, on substituting the explicit form of the matrix wave function $\Phi_{n}$, putting $m=0$, and, finally, on taking the integral over $p$ by means of the orthogonality condition (2.36), one finds this matrix element in the chiral limit to be

$$
A_{\mu}=\delta_{n \pi} \sqrt{\frac{N_{C}}{\pi}} P_{\mu} \frac{1}{\sqrt{2 P_{0}}} .
$$

Comparing expression (3.29) with the definition of the decay constant for the $n$th meson,

$$
A_{\mu}=f_{n} P_{\mu} \Psi_{n}(0),
$$

one can easily conclude that in the chiral limit

$$
f_{n}=\delta_{n \pi} \sqrt{\frac{N_{C}}{\pi}}
$$

that coincides with relation (2.80). 


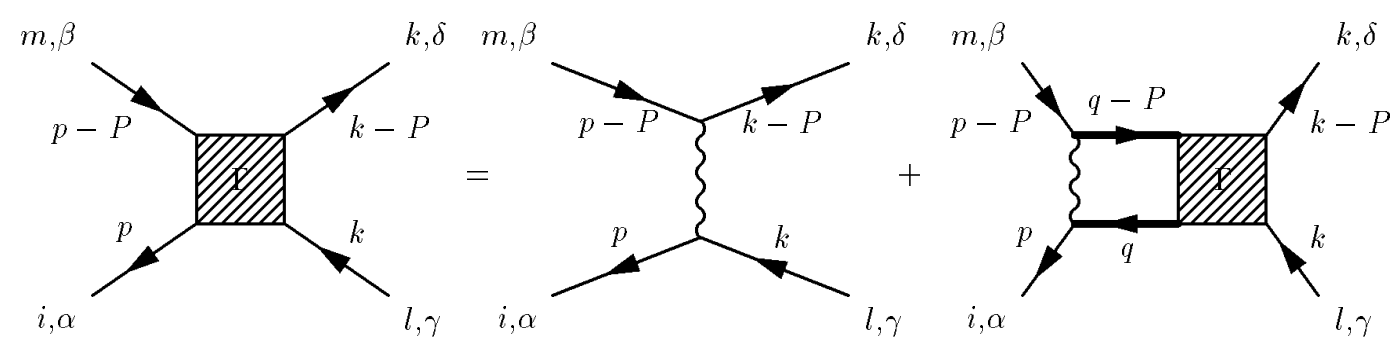

Figure 8: Graphical representation of the quark-quark scattering amplitude.

\subsection{Quark-quark scattering amplitude}

In this subsection we come to calculation of one of the most fundamental objects in the theory - the quark-quark scattering amplitude. If known, this object allows one to define the dressed current vertices and thus to investigate such properties of the theory as Ward identities, current conservation laws, etc. Equation for this object is given in the diagrammatic form in Fig.8 and reads

$$
\begin{gathered}
\Gamma_{i k, l m}^{\alpha \delta, \gamma \beta}\left(p, k, P_{\mu}\right)=-i g^{2}\left(\gamma_{0}\right)_{i l}\left(\gamma_{0}\right)_{k m} \frac{1}{(p-k)^{2}}\left(t^{a}\right)_{\gamma}^{\alpha}\left(t^{a}\right)_{\beta}^{\delta} \\
-i g^{2} \int \frac{d^{2} q}{(2 \pi)^{2}} \frac{1}{(p-q)^{2}}\left(\gamma_{0}\right)_{i s} S_{s t}(q) \Gamma_{t k, l n}^{\omega \delta, \gamma \sigma}\left(q, k, P_{\mu}\right) S_{n r}(q-P)\left(\gamma_{0}\right)_{r m}\left(t^{a}\right)_{\omega}^{\alpha}\left(t^{a}\right)_{\beta}^{\sigma},
\end{gathered}
$$

where Greek and Latin letters stand for the colour and the spinor indices, respectively.

Then one can separate the colour structure of $\Gamma$,

$$
\Gamma_{i k, l m}^{\alpha \delta, \gamma \beta}=\frac{1}{N_{C}} \delta_{\beta}^{\alpha} \delta_{\gamma}^{\delta} \Gamma_{i k, l m},
$$

and it is also convenient to introduce a new function $\tilde{\Gamma}$ defined as

$$
\tilde{\Gamma}_{s k, l r}\left(q_{\mu}, k, P_{\mu}\right)=S_{s t}(q) \Gamma_{t k, l n}\left(q, k, P_{\mu}\right) S_{n r}(q-P) .
$$

Due to the instantaneous type of the interaction induced by the two-dimensional gluon, it is also useful to define the amplitude $\Phi$ as an integral of $\tilde{\Gamma}$ over $q_{0}$,

$$
\int \frac{d q_{0}}{2 \pi} \tilde{\Gamma}_{s k, l r}\left(q_{\mu}, k, P_{\mu}\right)=\Phi_{s k, l r}\left(q, k, P_{\mu}\right) .
$$

Then the equation for the new function is

$$
\begin{gathered}
\Phi_{a k, l b}\left(p, k, P_{\mu}\right)=\frac{(2 \pi)^{2} \gamma}{(p-k)^{2}}\left(\gamma_{0}\right)_{i l}\left(\gamma_{0}\right)_{k m}\left[i \int \frac{d p_{0}}{2 \pi} S_{a i}(p) S_{m b}(p-P)\right] \\
+\gamma \int \frac{d q}{(p-q)^{2}}\left(\gamma_{0}\right)_{i s}\left(\gamma_{0}\right)_{r m} \Phi_{s k, l r}\left(q, k, P_{\mu}\right)\left[i \int \frac{d p_{0}}{2 \pi} S_{a i}(p) S_{m b}(p-P)\right] .
\end{gathered}
$$

The object in the square brackets can be easily integrated out using formula (A.1) and the result reads

$$
i \int \frac{d p_{0}}{2 \pi} S_{a i}(p) S_{m b}(p-P)=\frac{\left(\Lambda_{+}(p) \gamma_{0}\right)_{a i}\left(\Lambda_{-}(p-P) \gamma_{0}\right)_{m b}}{E(p)+E(P-p)-P_{0}}
$$




$$
+\frac{\left(\Lambda_{-}(p) \gamma_{0}\right)_{a i}\left(\Lambda_{+}(p-P) \gamma_{0}\right)_{m b}}{E(p)+E(P-p)+P_{0}}
$$

The simplest way to proceed further is to guess the general structure of the amplitude $\Phi$ to be

$$
\Phi_{s k, l r}\left(q, k, P_{\mu}\right)=\sum_{n=-\infty}^{\infty} \frac{\Phi_{s r}^{n}(q, P) \chi_{k l}^{n}(k, P)}{P_{0}-P_{0}^{n}},
$$

so that after some algebraic transformations with the use of the matrix bound-state equation (3.6) one arrives at

$$
\begin{gathered}
\sum_{n=-\infty}^{\infty} \chi_{k l}^{n}(k, P)\left[\frac{\Lambda_{+}(p) \Phi^{n}(p, P) \Lambda_{-}(P-p)}{E(p)+E(P-p)-P_{0}}-\frac{\Lambda_{-}(p) \Phi^{n}(p, P) \Lambda_{+}(P-p)}{E(p)+E(P-p)+P_{0}}\right]_{a b} \\
=\frac{(2 \pi)^{2} \gamma}{(p-k)^{2}}\left[\frac{\left(\Lambda_{+}(p)\right)_{a l}\left(\Lambda_{-}(P-p)\right)_{k b}}{E(p)+E(P-p)-P_{0}}-\frac{\left(\Lambda_{-}(p)\right)_{a l}\left(\Lambda_{+}(P-p)\right)_{k b}}{E(p)+E(P-p)+P_{0}}\right] .
\end{gathered}
$$

We parametrize the function $\chi^{n}(p, P)$ in the form:

$$
\chi^{n}(p, P)=2 \pi \gamma \int d q \frac{\psi^{n}(q, P)}{(p-q)^{2}},
$$

with $\psi^{n}(p, P)$ being a new unknown function. Substituting (3.40) into (3.39), we arrive at two simple equations defining $\psi^{n}(p, P)$,

$$
\sum_{n=-\infty}^{\infty} S p\left[\psi^{n}(q, P) \Phi_{ \pm}^{n}(p, P)\right]= \pm 2 \pi \delta(p-q) .
$$

With the help of the formulae from Appendix A the following solution to these equations can be found:

$$
\psi^{n}(p, P)=\operatorname{sign}(n) \Phi^{n+}(p, P),
$$

and, hence,

$$
\chi^{n}(p, P)=2 \pi \gamma \operatorname{sign}(n) \int d q \frac{\Phi^{n+}(q, P)}{(p-q)^{2}},
$$

that gives for the quark-quark scattering amplitude [35]:

$$
\begin{gathered}
\Gamma_{i k, l m}\left(p, k, P_{\mu}\right)=\frac{2 \pi i \gamma}{(p-k)^{2}}\left(\gamma_{0}\right)_{i l}\left(\gamma_{0}\right)_{k m}-i \gamma^{2} \sum_{n} \frac{1}{P_{0}-P_{0}^{n}}\left(\Gamma_{n}(p, P)\right)_{i m}\left(\bar{\Gamma}_{n}(k, P)\right)_{k l} \\
+i \gamma^{2} \sum_{n} \frac{1}{P_{0}+P_{0}^{n}}\left(\gamma_{0} \bar{\Gamma}_{n}(P-p, P) \gamma_{0}\right)_{i m}\left(\gamma_{0} \Gamma_{n}(P-k, P) \gamma_{0}\right)_{k l},
\end{gathered}
$$

where the sum over mesons $\sum_{n}$ counters only positive excitation numbers with $n>0$.

A couple of comments concerning the solution (3.44) is in order. First of all, note that, once the wave function $\Phi^{n}$ contains only symmetric matrices $\gamma_{0}$ and $\gamma_{5}$, then $\Phi_{a b}^{n}=\Phi_{b a}^{n}$ and, hence,

$$
\Gamma_{i k, l m}\left(p, k, P_{\mu}\right)=\Gamma_{m l, k i}\left(p, k, P_{\mu}\right) .
$$

The other comment concerns the form of the solution (3.44). One can see that the quarkquark scattering goes through the formation of a one-meson intermediate state and that the last two terms in (3.44) give nothing but the sum over the full mesonic propagators. 


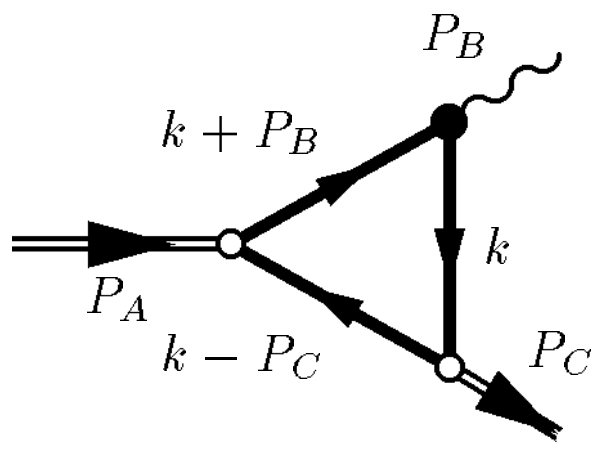

Figure 9: Graphical representation of the mesonic form factor.

Such way, we end with an effective diagrammatic technique which involves the dressed quark propagator (3.1) (see (A.1) for its ultimate form), the dressed meson-quark-antiquark amplitude ( $\Gamma$ for the incoming and $\bar{\Gamma}$ for the outgoing mesons given by (3.8)), the dressed quark-antiquark scattering amplitude (3.44) and the constant $-i \gamma / \sqrt{N_{C}}$ which is to be inserted into every vertex where a meson couples to a quark-antiquark pair. In addition, each quark loop brings two extra factors, the standard fermionic (-1) and $N_{C}$ from the colour trace. One can use these ingredients as bricks for building any hadronic process in the theory.

\subsection{Vector and axial-vector currents conservation. Ward identi- ties.}

As stated in the previous subsection, with the quark-quark scattering amplitude in hands we are in the position to study properties of currents in the 't Hooft model. Let us prove the currents conservation first. For the vector current we are interested in the matrix element

$$
V_{\mu}^{M}(P)=\left\langle\Omega\left|\bar{q} \gamma_{\mu} q\right| M, P\right\rangle,
$$

depicted in Fig. Ø)(a).

On writing the corresponding matrix element and performing the integration over the energy $p_{0}$, one finds:

$$
V_{\mu}^{M}=i \gamma \sqrt{N_{C}} \int \frac{d^{2} p}{(2 \pi)^{2}} S p\left[S(p-P) \gamma_{\mu} S(p) \Gamma_{M}(p, P)\right]=\sqrt{N_{C}} \int \frac{d p}{2 \pi} S p\left[\gamma_{\mu} \Phi_{M}(p, P)\right] .
$$

It is easy to check that, multiplying the bound-state equation (3.6) by $\gamma_{0} \sqrt{N_{C}} / 2 \pi$, taking trace over the spinor indices, and integrating all terms of the resulting equation over the momentum $p$, one arrives at the relation:

$$
P_{0}^{M}\left[\sqrt{N_{C}} \int \frac{d p}{2 \pi} S p\left[\gamma_{0} \Phi_{M}(p, P)\right]\right]-P\left[\sqrt{N_{C}} \int \frac{d p}{2 \pi} S p\left[\gamma_{1} \Phi_{M}(p, P)\right]\right]=0 .
$$

Combining it with the definition (3.47), one finds that the vector current is conserved,

$$
P_{0}^{M} V_{0}^{M}-P V^{M}=0 .
$$

Similarly, defining the axial-vector current as

$$
A_{\mu}^{M}(P)=\left\langle\Omega\left|\bar{q} \gamma_{\mu} \gamma_{5} q\right| M, P\right\rangle
$$



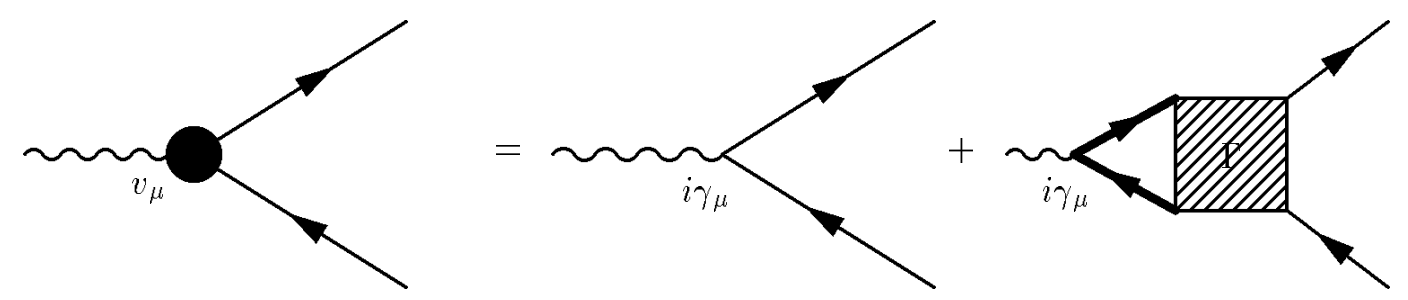

Figure 10: Graphical representation of the dressed vector current-quark-antiquark vertex.

i.e., using the diagram (b) in Fig.7 and performing the same steps concerning the boundstate equation as before, but with the evident change $\gamma_{0} \rightarrow \gamma_{1}$ in the multiplier, one can derive the axial-vector current divergency in the form:

$$
P_{0}^{M} A_{0}^{M}-P A^{M}=-2 m \sqrt{N_{C}} \int \frac{d k}{2 \pi} S p\left[\gamma_{5} \Phi_{M}\right],
$$

which turns into the axial-vector-current conservation law in the chiral limit.

It is instructive to see how the same relations appear in the Hamiltonian approach. We shall concentrate only on the vector current conservation law as a similar analysis for the axial-vector current is straightforward then.

We start from the definition of the vector current,

$$
J_{\mu}(x)=\bar{q}(x) \gamma_{\mu} q(x)
$$

and reformulate it in terms of mesonic creation and annihilation operators, 巴尸

$$
\begin{gathered}
J_{0}(x)=2 \sqrt{N_{C}} \int \frac{d P}{2 \pi} e^{i P x} \sum_{n} e^{-i P_{0}^{n} x_{0}} m_{n}(P) \int \frac{d p}{2 \pi} g_{n}(p, P) f_{0}(p, P)+h . c ., \\
J(x)=2 \sqrt{N_{C}} \int \frac{d P}{2 \pi} e^{i P x} \sum_{n} e^{-i P_{0}^{n} x_{0}} m_{n}(P) \int \frac{d p}{2 \pi} f_{n}(p, P) g_{0}(p, P)+h . c .,
\end{gathered}
$$

where $f_{n}(p, P)$ and $g_{n}(p, P)$ are introduced in (2.86), whereas $f_{0}$ and $g_{0}$ are other notations for the pion wave functions $f_{\pi}$ and $g_{\pi}$.

Then calculating the corresponding matrix element explicitly one finds:

$$
\begin{aligned}
& P_{0}^{M} V_{0}^{M}-P V^{M}=i\left\langle\Omega\left|\partial_{\mu} J_{\mu}(0)\right| M, P\right\rangle= \\
& =2 \sqrt{N_{C}} \int \frac{d p}{2 \pi}\left[P_{0}^{M} g_{M}(p, P) f_{0}(p, P)-P f_{M}(p, P) g_{0}(p, P)\right]=0,
\end{aligned}
$$

where the r.h.s. of this equation vanishes due to the bound-state equation (2.40) or (3.6).

Now we turn to the investigation of the mesonic form-factors defined by the diagram depicted in Fig.9, but we need to know the dressed current-quark-antiquark vertices first.

Let us start from the vector current. The corresponding expression for the diagrams given in Fig.10 reads

$$
\left(v_{\mu}^{k l}(p, P)\right)_{\beta}^{\alpha}=i\left(\gamma_{\mu}\right)_{k l} \delta_{\beta}^{\alpha}-\int \frac{d^{2} q}{(2 \pi)^{2}} S_{d a}(q) i\left(\gamma_{\mu}\right)_{a b} \delta_{\delta}^{\gamma} S_{b c}(q-P) \Gamma_{c k, l d}^{\delta \alpha, \beta \gamma}(q, p, P)=v_{\mu}^{k l} \delta_{\beta}^{\alpha},
$$

\footnotetext{
${ }^{11}$ Note that we have restored the explicit dependence of operators $m_{n}$ on time in the form $m_{n}\left(x_{0}, P\right)=$ $e^{-i P_{0}^{n} x_{0}} m_{n}(P)$ with $P_{0}^{n}$ being the energy of the $n$th mesonic state moving with the total momentum $P$.
} 
where, as before, the colour indices are denoted by the Greek symbols, whereas Latin ones stand for the spinor indices. Substituting the explicit form of the quark-quark scattering amplitude $\Gamma_{c k, l d}^{\delta \alpha, \beta \gamma}(q, p, P)$ from (3.44) into (3.56), one finds [35]:

$$
\begin{aligned}
& v_{\mu}\left(p, P_{\mu}\right)=i \gamma_{\mu}+i \gamma \sum_{n} \frac{\bar{\Gamma}_{n}(p, P)}{P_{0}-P_{0}^{n}} \int \frac{d q}{2 \pi} S p\left[\gamma_{\mu} \Phi_{n}(q, P)\right] \\
& -i \gamma \sum_{n} \frac{\gamma_{0} \Gamma_{n}(P-p, P) \gamma_{0}}{P_{0}+P_{0}^{n}} \int \frac{d q}{2 \pi} S p\left[\gamma_{\mu} \Phi_{n}^{+}(P-q, P)\right] .
\end{aligned}
$$

After tedious but straightforward calculations using the explicit form of the matrix bound-state equation (3.6) and the matrix wave function (3.7) the following relation can be proved:

$$
-i P_{\mu} v_{\mu}(p, P)=S^{-1}(p)-S^{-1}(p-P),
$$

which is nothing but the vector Ward identity for the 't Hooft model (similar expression in the light-cone gauge was derived in [4]).

Now it is a simple task to substitute the solution (3.58) into the matrix element written for the diagram in Fig.9 with the vector current instead of the curly line and to arrive at the vector current conservation law,

$$
Q_{\mu}\left\langle M, P\left|v_{\mu}\right| M^{\prime}, P^{\prime}\right\rangle=0, \quad Q_{\mu}=P_{\mu}-P_{\mu}^{\prime} .
$$

Similar calculations give the following results for the axial-vector current in the chiral limit 35],

$$
\begin{gathered}
a_{\mu}\left(p, P_{\mu}\right)=i \gamma_{\mu} \gamma_{5}+i \gamma \sum_{n} \frac{\bar{\Gamma}_{n}(p, P)}{P_{0}-P_{0}^{n}} \int \frac{d q}{2 \pi} S p\left[\gamma_{\mu} \gamma_{5} \Phi_{n}(q, P)\right] \\
-i \gamma \sum_{n} \frac{\gamma_{0} \Gamma_{n}(P-p, P) \gamma_{0}}{P_{0}+P_{0}^{n}} \int \frac{d q}{2 \pi} S p\left[\gamma_{\mu} \gamma_{5} \Phi_{n}^{+}(P-q, P)\right] \\
-i P_{\mu} a_{\mu}(p, P)=S^{-1}(p) \gamma_{5}+\gamma_{5} S^{-1}(p-P)
\end{gathered}
$$

and, finally,

$$
Q_{\mu}\left\langle M, P\left|a_{\mu}\right| M^{\prime}, P^{\prime}\right\rangle=0, \quad Q_{\mu}=P_{\mu}-P_{\mu}^{\prime} .
$$

Relation (3.61) plays the role of the axial-vector Ward identity.

In conclusion let us notice that one could arrive at the same results using the Hamiltonian approach but at the price of a much more complicated algebra and a much less transparent interpretation of the results in terms of Feynman diagrams.

\subsection{The pionic vertex}

The general structure of the mesonic vertex can be considerably simplified in case of the pion, since the explicit form of the pionic wave function is known. Indeed, substituting the solution (2.45) into the matrix form (3.7) and then into the definition (3.8), one easily finds [35]:

$$
\Gamma_{\pi}(p, P)=S^{-1}(p)\left(1+\gamma_{5}\right)-\left(1-\gamma_{5}\right) S^{-1}(p-P) .
$$

It is also instructive to compare formula (3.63) with the Ward identities for the vector and axial-vector currents derived in the previous subsection, equations (3.58) and (3.61), respectively. As a result, one finds the following relation for the pionic vertex [35]:

$$
\Gamma_{\pi}(p, P)=-i P_{\mu} v_{\mu}(p, P)-i P_{\mu} a_{\mu}(p, P) .
$$


Note that it is not surprise that the pion couples not only to the axial-vector, but to the vector current as well. The reason is that in the two-dimensional theory the axial-vector and the vector currents are dual to one another,

$$
J_{\mu}^{5}(x)=\varepsilon_{\mu \nu} J^{\nu}(x),
$$

where $\varepsilon_{\mu \nu}$ is the totally-antisymmetric Levy-Civita tensor in two dimensions.

\section{Strong decays}

This section is devoted to investigation of hadronic processes in the 't Hooft model at the example of the decay $A \rightarrow B+C$. In subsections 4.1 and 4.2 we derive the amplitude of such a decay using the Hamiltonian and matrix approach, respectively. We discuss its properties and correspondence with nonrelativistic models. Subsection 4.3 is devoted to derivation and justification of the two-dimensional Adler selfconsistency condition ("Adler zero").

\subsection{Suppressed terms in the Hamiltonian}

In section 2 we developed the Hamiltonian approach to $\mathrm{QCD}_{2}$ and diagonalized the Hamiltonian of the model in the mesonic sector. Now let us turn to corrections to the Hamiltonian (2.39) suppressed by powers of $N_{C}$ in the denominator. The leading correction is of order $O\left(1 / \sqrt{N_{C}}\right)$ and it comes from the terms containing the products $M B, M D, M^{+} B$, and $M^{+} D$ of the operators introduced in (2.25),

$$
\begin{array}{r}
\Delta H=-\frac{\gamma}{\sqrt{N_{C}}} \int \frac{d p d k d q d Q}{(2 \pi)^{3}(p-k)^{2}} \cos \frac{\theta(p)-\theta(k)}{2} \sin \frac{\theta(Q-p)-\theta(Q-k)}{2} \\
\times\left[M^{+}(p, p-Q) M^{+}(k-Q, q) M(k, q)+M^{+}(p-Q, p) M^{+}(q, k-Q) M(q, k)\right. \\
\left.-M^{+}(q, p) M(q, p-Q) M(k-Q, k)-M^{+}(p, q) M(p-Q, q) M(k, k-Q)\right],
\end{array}
$$

where $M$ and $M^{+}$can be defined through the meson creation and annihilation operators (see also (2.35) ),

$$
\begin{aligned}
M^{+}(p, k) & =\sum_{n=0}^{\infty}\left[m_{n}^{+}(k-p) \varphi_{+}^{n}(k, k-p)-m_{n}(p-k) \varphi_{+}^{n}(p, p-k)\right], \\
M(p, k) & =\sum_{n=0}^{\infty}\left[m_{n}(k-p) \varphi_{+}^{n}(k, k-p)-m_{n}^{+}(p-k) \varphi_{+}^{n}(p, p-k)\right] .
\end{aligned}
$$

As easily seen from (4.1), this correction describes vertices with three mesons involved.

\section{2 $\quad A \rightarrow B+C$ decay amplitude}

Now we are in the position to study the hadronic processes in the 't Hooft model. Strong decays $A \rightarrow B+C$ are of most interest for us [35]. As mentioned before, in the leading order in $N_{C}$ the 't Hooft model describes free mesons (see (2.39)), whereas the interaction between them is hidden in the suppressed terms partially restored in the previous subsection. Thus we expect the amplitude $M(A \rightarrow B+C)$ to be of order $1 / \sqrt{N_{C}}$, whereas $M(A+B \rightarrow$ 
$C+D) \sim 1 / N_{C}$. In this subsection we study the influence of the backward motion described

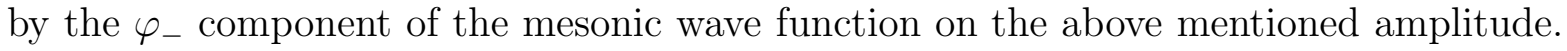

The Hamiltonian approach developed before gives the most straightforward way to calculate the amplitude of the strong decay since one just needs to evaluate the following matrix element:

$$
M(A \rightarrow B+C)=\left\langle B\left(P_{B}\right) C\left(P_{C}\right)|H+\Delta H| A\left(P_{A}\right)\right\rangle=\left\langle B\left(P_{B}\right) C\left(P_{C}\right)|\Delta H| A\left(P_{A}\right)\right\rangle .
$$

With the help of the explicit form of the operator $\Delta H$ given in (4.1) one easily finds the general form of the amplitude in terms of mesonic wave functions in the rest frame of the decaying particle $A\left(P_{A}=0, P_{B}=-P_{C}=p\right)$ to be (see also [36] where a six-term decay amplitude is discussed):

$$
\begin{gathered}
M(A \rightarrow B+C)= \\
\frac{\gamma}{\sqrt{N_{C}}} \int \frac{d k d q}{(q-k)^{2}}\left\{-\varphi_{-}^{A}(k+p, 0) \varphi_{-}^{B}(k+p, 0)\left[c(-p, q, k) \varphi_{+}^{C}(q,-p)+s(-p, q, k) \varphi_{-}\right]\right. \\
-\varphi_{+}^{A}(k+p, 0) \varphi_{+}^{C}(k,-p)\left[c(p, q+p, k+p) \varphi_{+}^{B}(q+p, p)+s(p, q+p, k+p) \varphi_{-}^{B}(q+p, p)\right] \\
-\varphi_{+}^{C}(k,-p) \varphi_{-}^{B}(k+p, p)\left[s(0, q+p, k+p) \varphi_{+}^{A}(q+p, 0)+c(0, q+p, k+p) \varphi_{-}^{A}(q+p, 0)\right] \\
+\varphi_{-}^{C}(k,-p) \varphi_{+}^{B}(k+p, p)\left[c(0, q+p, k+p) \varphi_{+}^{A}(q+p, 0)+s(0, q+p, k+p) \varphi_{-}^{A}(q+p, 0)\right] \\
+\varphi_{-}^{A}(k+p, 0) \varphi_{-}^{C}(k,-p)\left[s(p, q+p, k+p) \varphi_{+}^{B}(q+p, p)+c(p, q+p, k+p) \varphi_{-}^{B}(q+p, p)\right] \\
\left.+\varphi_{+}^{A}(k+p, 0) \varphi_{+}^{B}(k+p, p)\left[s(-p, q, k) \varphi_{+}^{C}(q,-p)+c(-p, q, k) \varphi_{-}^{C}(q,-p)\right]\right\} \\
+(B \leftrightarrow C, p \leftrightarrow-p),
\end{gathered}
$$

where

$$
\begin{aligned}
& c(p, q, k)=\cos \frac{\theta(k)-\theta(q)}{2} \sin \frac{\theta(p-k)-\theta(p-q)}{2} \\
& s(p, q, k)=\sin \frac{\theta(k)-\theta(q)}{2} \cos \frac{\theta(p-k)-\theta(p-q)}{2} .
\end{aligned}
$$

In spite of its frightening appearance, the amplitude (4.4) has a very simple structure. Indeed, it contains six terms, i.e., three times more than one could naively expected and this is entirely due to the presence of the $\varphi_{-}$component in the mesonic wave function. If one neglects the backward motion contributions in the amplitude (4.4) and inserts the nonrelativistic values of the angle $\theta(\cos \theta(p)=1, \sin \theta(p)=p / m)$, then it reproduces the standard quark-model decay amplitude due to the OGE Coulomb interaction [37] adapted to the two-dimensional case. It is clear, however, that the substitution of the nonrelativistic angle is not justified for kinematical reasons.

One can arrive at exactly the same expression for the amplitude using the matrix approach and writing the matrix element for the diagrams depicted in Fig.11,

$$
\begin{gathered}
M(A \rightarrow B+C)= \\
-\frac{i \gamma^{3}}{\sqrt{N_{C}}} \int \frac{d^{2} k}{(2 \pi)^{2}} S p\left[\Gamma_{A}\left(k+P_{B}, P_{A}\right) S\left(k-P_{C}\right) \bar{\Gamma}_{C}\left(k, P_{C}\right) S(k) \bar{\Gamma}_{B}\left(k+P_{B}, P_{B}\right) S\left(k+P_{B}\right)\right] \\
+(B \leftrightarrow C) .
\end{gathered}
$$

In the meantime, the matrix approach proves more convenient in studies of the decay amplitude (4.4), 4.5), so we stick with it in the next subsection considering pions in the final state. 


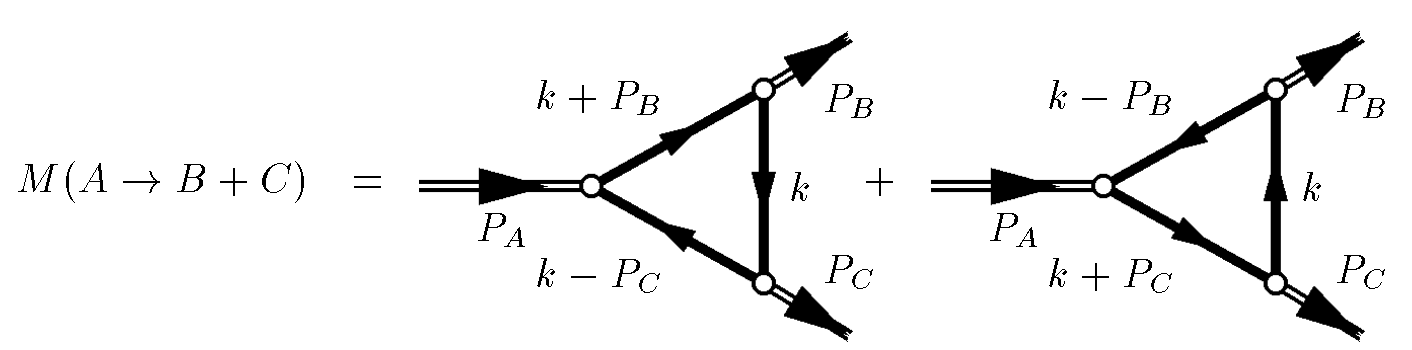

Figure 11: Graphical representation of the amplitude for the decay $A \rightarrow B+C$.

\subsection{Adler selfconsistency condition}

In this subsection we have one more careful look at the pions - namely, at their role in the hadronic processes. We remind the reader that these are pions to suffer most drastically from the presence of the backward motion of the $q \bar{q}$ pair described by the $\varphi_{-}$component of the wave function. On the other hand, hadronic processes with the pions in the final state are much better investigated experimentally, so that any theoretical hint as to how the chiral nature of the pion affects hadronic decays is of paramount importance. The 't Hooft model for two-dimensional QCD is a source of such hints.

Thus let the meson $B$ be the pion. We substitute the explicit form of the pionic vertex (3.63) into expression (4.5) and after simple algebraic exercises arrive at a striking conclusion that 35]

$$
M(A \rightarrow \pi+C) \equiv 0
$$

in the chiral limit. Note that this result could be anticipated in view of the identification (3.64) and the current conservation laws (3.59) and (3.62). The condition (4.6) is nothing but the two-dimensional analogue of the celebrated Adler selfconsistency condition for amplitudes with soft pions involved [38]. It is not surprise, that it holds true for any value of the pion momentum as, in view of the dimensionlessness of the pion decay constant $f_{\pi}$, there is no soft scale in the model which could play the role of the edge for this condition.

Let us conclude with a couple of comments concerning the condition (4.6). First of all, the pion turns out sterile, at least in the subleading order in $N_{C}$. On the other hand, applying the above qualitative analysis concerning the dimension of $f_{\pi}$, one can extend the condition (4.6) to the case of any hadronic process with pions involved, so that the pion is completely decoupled from the spectrum in the chiral limit in all orders in $N_{C}$.

The formal reason for the condition (4.6) is a totally destructive interference between the $\varphi_{+}$and the $\varphi_{-}$components of the pionic wave function. They were found to be of the same order of magnitude and, hence, all six terms of the amplitude (4.4) are equally important in establishing the condition (4.6). The latter observation automatically invalidates any attempts to describe the pion in the framework of a constituent quark model when the $\varphi_{-}$ component is completely lost. It seems quite unprobable that the above drawback of the quark models in $\mathrm{QCD}_{2}$, as well as in $\mathrm{QCD}_{4}$, could be cured by simple prescriptions, like multiplying the naive 2-term amplitude by extra "magic" factors [36] or whatever. 


\section{Conclusions and prospectives}

Phenomenological successes of quark models do tell us that the constituent quarks are the correct degrees of freedom in the nonperturbative domain. In these models the lowest ${ }^{1} S_{0} q \bar{q}$ state exists on the same footing as other mesons. As it was already mentioned, there are no direct indications that the confinement and the chiral symmetry breaking are interrelated in case of $\mathrm{QCD}_{4}$. Nevertheless, if such a scenario does not take place, then one easily runs into troubles with constituent models: two pions exist, one is a quark bound state, and the other one is responsible for the chiral symmetry breaking. Of course, a roundabout way is to disregard quark models completely. However, it is more economical to organize the confinement and the chiral symmetry breaking due to one and the same mechanism.

Developing such an approach for $\mathrm{QCD}_{4}$ is not a straightforward task. Models [15, 16] incorporate the main ingredients, the gap equation similar to (2.13) and the Bethe-Salpeter equation similar to (2.40). The existence of the chirally-noninvariant solution of the gap equation implies the existence of the Goldstone boson as the lowest $q \bar{q}$ state. The $\varphi_{+}$and

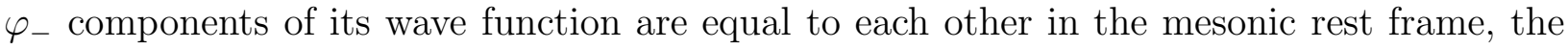
axial-vector current is conserved in the chiral limit, and all the relations of the current algebra are satisfied. In the meantime, the role of the $\varphi_{-}$component of the wave functions for all other mesons is less drastic, and parity degeneracy is restored for higher quarkonia [15].

Unfortunately, all these nice features persist at the price of confining interaction employed, chosen as the time component of a vector force. First, such a model is not covariant, that prevents from proceeding further along the lines described above. In particular, one cannot make Lorentz boosts within this model and cannot describe the strong decays. Another drawback is even more important - namely, the interaction is not compatible with the area law for the isolated Wilson loop. This point holds true not only for the model [15] where the interaction potential is the three-dimensional oscillator one. It is also so for rather sophisticated models with linear confinement too (see, e.g., [39]). The reason is that the area law yields linear confining potential only for heavy constituents. Besides that, the last, but not the least, objection is the lack of the gauge invariance in such kind of models.

An approach suggested in [17] is rather promising in all these respects. The confining interaction employed there is given by a set of gluonic field-strength correlators, which produce the area law. These correlators are Lorentz covariant by construction, and gauge invariance is preserved too. The latter is ensured by using the generalized Fock-Schwinger gauge (Balitsky gauge), which leads to the gluonic correlators explicitly dependent on the reference contour and, as a consequence, explicitly translationally noninvariant. A simple two-dimensional example of such a correlator is given by the expression (2.55). The problem is very technically involved due to this fact, but at the same time it is very physically transparent. Indeed, the interaction of such a kind describes the string which is developed between the constituents (for the recent progress in this direction see [40]). On the other hand, the same correlators are responsible for the chiral condensate formation [17]. We expect that the quark model followed from such a formalism could be able to describe $q \bar{q}$ bound states including the pion.

In conclusion, we state once more that a reasonable model in four dimensions is welcome in order to find the pionic solution playing the twofold role: being a true $q \bar{q}$ state it is also the Goldstone boson. In our opinion the two-dimensional 't Hooft model gives a brilliant example of how this could happen in Nature. 
The authors would like to thank Yu.A.Simonov for encouraging them to write this review and for stimulating discussions, P.Bicudo and J.E.Ribeiro for the help in studying the vacuum properties of the theory and many useful and enlightening discussions, as well as P.Maris for reading the manuscript and critical remarks. They are also grateful to the staff of the Centro de Física das Interacções Fundamentais (CFIF-IST) for cordial hospitality during their stay in Lisbon.

Financial support of RFFI grants 00-02-17836 and 00-15-96786, INTAS-RFFI grant IR97-232 and INTAS CALL 2000, project \# 110 is gratefully acknowledged. One of the authors (A.N.) is also supported by RFFI grant 01-02-06273.

\section{Appendix A}

In this appendix we collect some formulae useful for the matrix approach.

The dressed quark Green's function (3.1) can be rewritten in a more convenient form if the projectors (2.11) are introduced,

$$
S\left(p_{\mu}\right)=\frac{\Lambda_{+}(p) \gamma_{0}}{p_{0}-E(p)+i \varepsilon}+\frac{\Lambda_{-}(p) \gamma_{0}}{p_{0}+E(p)-i \varepsilon} .
$$

As mentioned before, one should be careful with the sign of the dispersive law $E(p)$ and keep the product $\varepsilon E(p)$, when combining the two fractions in (A.1) together [2]. The propagator (A.1) contains all radiative corrections and should be assigned to the internal quark lines (fat lines in diagrams).

We find it useful to split the matrix wave function (3.7) into two pieces by means of the projectors (2.11), ए]

$$
\Phi_{\eta}(p, P)=\Lambda_{\eta}(p) \Phi(p, P) \Lambda_{\bar{\eta}}(P-p), \quad \Phi(p, P)=\Phi_{\eta}(p, P)+\Phi_{\bar{\eta}}(p, P),
$$

where either $\eta=+, \bar{\eta}=-$, or, vice versa, $\eta=-, \bar{\eta}=+$. Then the completeness condition for $\Phi$ 's reads

$$
\sum_{n} S p\left[\Phi_{+}^{n+}(p, P) \Phi_{+}^{n}(q, P)-\Phi_{-}^{n+}(p, P) \Phi_{-}^{n}(q, P)\right]=2 \pi \delta(p-q)
$$

which can be easily derived, using the corresponding properties of the functions $\varphi_{ \pm}$and the relation

$$
\begin{gathered}
S p\left[\Phi_{\eta}^{n+}(p, P) \Phi_{\eta^{\prime}}^{m}(q, P)\right]=\varphi_{\eta}^{n}(p, P) \varphi_{\eta^{\prime}}^{m}(q, P)\left[\delta_{\eta \eta^{\prime}} \cos \frac{\theta(p)-\theta(q)}{2} \cos \frac{\theta(P-p)-\theta(P-q)}{2}\right. \\
\left.-\delta_{\eta \bar{\eta}^{\prime}} \sin \frac{\theta(p)-\theta(q)}{2} \sin \frac{\theta(P-p)-\theta(P-q)}{2}\right] .
\end{gathered}
$$

We also give two formulae useful for the derivation of the quark-quark scattering amplitude,

$$
\begin{gathered}
\Phi_{\eta}^{-n}(p, P)=\eta_{n} \Phi_{\bar{\eta}}^{n+}(P-p, P), \\
S p\left[\Phi^{n+}(P-p, P) \Phi_{\eta}^{m}(P-q, P)\right]=\eta_{n} \eta_{m} S p\left[\Phi^{n+}(p, P) \Phi_{\eta}^{m}(q, P)\right],
\end{gathered}
$$

\footnotetext{
${ }^{12}$ In this appendix we suppress the index numerating the mesonic states, keeping it only when necessary.
} 
where $\eta$ and $\bar{\eta}$ are defined above and $\eta_{n}$ is connected to the spatial parity of the state (see equation (2.44)).

The next two formulae come out directly from the bound-state equation (2.40),

$$
\begin{gathered}
\Lambda_{\eta}(p) \gamma_{0} \Gamma(p, P) \Lambda_{\bar{\eta}}(p-P) \gamma_{0}=\frac{1}{\gamma}\left[E(p)+E(P-p)-\eta P_{0}\right] \Lambda_{\eta}(p) \Phi(p, P) \Lambda_{\bar{\eta}}(p-P) \\
=\frac{1}{\gamma}\left[E(p)+E(P-p)-\eta P_{0}\right] \Phi_{\eta}(p, P) \\
\Lambda_{\eta}(p-P) \gamma_{0} \bar{\Gamma}(p, P) \Lambda_{\bar{\eta}}(p) \gamma_{0}=\frac{1}{\gamma}\left[E(p)+E(P-p)+\eta P_{0}\right] \Lambda_{\eta}(p-P) \gamma_{0} \Phi^{+}(p, P) \Lambda_{\bar{\eta}}(p) \gamma_{0} \\
=\frac{1}{\gamma}\left[E(p)+E(P-p)+\eta P_{0}\right] \gamma_{0} \Phi_{\bar{\eta}}^{+}(p, P) \gamma_{0},
\end{gathered}
$$

whereas with the help of relations (2.43) and (2.44) one finds

$$
\Gamma_{n}(P-p, P)=\eta_{n} \gamma_{0} \bar{\Gamma}_{-n}(p, P) \gamma_{0}
$$

\section{References}

[1] G.'t Hooft, Nucl.Phys. B75, 461 (1974)

[2] I.Bars and M.B.Green, Phys.Rev. D17, 537 (1978)

[3] C.J.Callan, N.Coote D.J.Gross, Phys.Rev. D13, 1649 (1976)

[4] M.B.Einhorn, Phys.Rev. D14, 3451 (1976)

[5] Ming Li, Phys.Rev. D34, 3888 (1986)

Ming Li, L.Wilets and M.C.Birse, J.Phys. G13, 915 (1987)

[6] A.R.Zhitnitskii, Sov.J.Nucl.Phys. 43, 999 (1986); 44, 139 (1986); Phys.Rev. D53, 5821 (1996)

[7] M.Burkardt, Phys.Rev. D53, 933 (1996)

[8] F.Lenz, M.Thies, S.Levit and K.Yazaki, Ann.Phys.(N.Y) 208, 1 (1991)

[9] B.Blok, M.Shifman and Da-Xin Zhang, Phys.Rev. D57, 2691 (1998)

[10] I.Bigi, M.Shifman, N.Uraltsev and A.Vainstein, Phys.Rev. D59, 054011 (1999)

[11] K.Hornbostel, S.J.Brodsky and H.C.Pauli D41, 3814 (1990)

[12] S.Dalley, I.R.Klebanov, Phys.Rev. D47, 2517 (1993)

[13] V.Vento, nucl-th/0010014, to appear in Proc. of the XVII European Conference on Few-Body Problems in Physics

[14] F.Karsch, hep-lat/9903031, in Copenhagen 1998, Strong and electroweak matter, p.101 
[15] A.Le Yaouanc, L.Oliver, O.Pene and J.-C.Raynal, Phys. Rev. D29, 1233 (1984);

A.Le Yaouanc, L.Oliver, S.Ono, O.Pene and J.-C.Raynal, Phys.Rev. D31, 137 (1985)

[16] P.Bicudo and J.E.Ribeiro, Phys.Rev. D42, 1611 (1990); ibid. 1625 (1990); ibid. 1635 (1990);

P.Bicudo, Phys.Rev. C60, 035209 (1999)

[17] Yu.A.Simonov, Phys.Atom.Nucl. 60, 2069 (1997)

[18] E.Witten, Nucl.Phys. B145, 110 (1978)

[19] S.Coleman, Commun.Math.Phys. 31, 259 (1973)

[20] K.Kikkawa, Ann.Phys. 66, 3633 (1981);

A.Nakamura and K.Odaka, Phys.Lett. B105, 392 (1981); Nucl.Phys. B202, 457 (1982);

S.G.Rajeev, Int.Journ.Mod.Phys. A9, 5583 (1994);

A.Dhar, C.Mandal and S.R.Wadia, Phys.Lett. B329, 15 (1994);

A.Dhar et.al. Int.Journ.Mod.Phys. A10, 15 (1995);

M.Cavicchi, Int.Journ.Mod.Phys. A10, 167 (1995);

K.Itakura, Phys.Rev. D54, 2853 (1996)

[21] H.G.Dosch, Phys.Lett. B190, 177 (1987);

H.G.Dosch, Yu.A.Simonov, Phys.Lett. B205, 339 (1988);

Yu.A.Simonov, Nucl.Phys. B307, 512 (1988);

Yu.A.Simonov, Yad.Fiz. 54, 192 (1991)

[22] A.Di Giacomo, H.G.Dosch, V.I.Shevchenko, Yu.A.Simonov, hep-ph/0007223

[23] P.Bicudo, A.V.Nefediev, J.E.Ribeiro, in preparation

[24] D.Gross, A.Neveu, Phys.Rev. D10, 3235 (1974)

[25] P.Rembiesa, Phys.Rev. D24, 1647 (1981);

I.Ojima, R.Fukada, Prog.Theor.Phys. 57, 1720 (1977)

[26] Yu.S.Kalashnikova, A.V.Nefediev A.V.Volodin, Phys.At.Nucl. 63, 1710 (2000)

[27] I.Bars, A.J.Hanson, Phys.Rev. D13, 1744 (1976);

W.A.Bardeen, I.Bars, A.J.Hanson, R.D.Peccei, Phys.Rev. D13, 2364 (1976);

Yu.S.Kalashnikova, A.V.Nefediev Phys.Lett. B399, 274 (1997)

[28] Yu.S.Kalashnikova, A.V.Nefediev, Phys.At.Nucl. 62, 323 (1999)

[29] I.I.Balitsky, Nucl.Phys. B254, 166 (1985)

[30] A.V.Nefediev, Talk given at 3rd International Conference in Quark Confinement and Hadron Spectrum (Confinement III), Newport News, VA, 7-12 Jun 1998; hep$\mathrm{ph} / 9808208$

[31] V.I.Shevchenko, Yu.A.Simonov, Phys.Rev.Lett. 85, 1811 (2000)

[32] N.Brambilla and A.Vairo, Phys.Lett. B407, 167 (1997) 
[33] Yu.S.Kalashnikova, A.V.Nefediev, Phys.Lett. B414, 149 (1997)

[34] M.Gell-Mann, R.J.Oakes and B.Renner, Phys.Rev. 175, 2195 (1968)

[35] Yu.S.Kalashnikova, A.V.Nefediev, Phys.Lett. B487, 371 (2000)

[36] E.S.Swanson, In Proc. of the CMU/JLab Workshop on Physics with High Energy Photons, Jeffrerson Lab (1998)

[37] E.S.Ackleh, T.Barnes, E.S.Swanson, Phys.Rev. D54, 6811 (1996)

[38] S.L.Adler, Phys. Rev. 139B, 1638 (1965)

[39] A.P.Szczepaniak, E.S.Swanson, Phys.Rev. D55, 1578 (1997)

F.J.Llanes-Estrada, S.R.Cotanch, hep-ph/0101078, in press

[40] Yu.A.Simonov, J.A.Tjon, Phys.Rev. 62, 014501 (2000); Phys.Rev. 62, 094511 (2000) 